\title{
1 4D electrical resistivity tomography (ERT) for aquifer thermal energy storage monitoring
}

3 Lesparre Nolwenn ${ }^{1,2}$, Robert Tanguy ${ }^{3,4,5}$, Nguyen Frédéric ${ }^{1}$, Boyle Alistair ${ }^{6}$, Hermans Thomas ${ }^{1,4,7}$

$4 \quad{ }^{1}$ Urban and Environmental Engineering, Applied Geophysics, Liege University, Quartier Polytech

5 1, Building B52/3, Allée de la découverte, 9, B-4000 Liege, Belgium, lesparre@unistra.fr,

6 f.nguyen@uliege.be

$7{ }^{2}$ now at: Laboratoire d'Hydrologie et Géochimie de Strasbourg, University of 8 Strasbourg/EOST/ENGEES, CNRS UMR 7517, 1 Rue Blessig, 67084 Strasbourg, France

$9{ }^{3}$ previously at: R\&D Department, AQUALE SPRL, Rue Montellier 22, B-5380 Noville-les-Bois, 10 Belgium

$11{ }^{4}$ F.R.S.-FNRS (Fonds de la Recherche Scientifique), Rue d'Egmont, 5, B-1000 Brussels, Belgium

$12{ }^{5}$ Urban and Environmental Engineering, Hydrogeology \& Environmental Geology, Liege University,

13 Quartier Polytech 1, Building B52/3, Allée de la découverte, 9, B-4000 Liege, 14 tanguy.robert@uliege.be

$15{ }^{6}$ School of Electrical Engineering and Computer Science, University of Ottawa, 800 King Edward 16 Avenue, Ottawa, Ontario, K1N 6N5, Canada, aboyle2@uottawa.ca

$17{ }^{7}$ Ghent University, Department of Geology, Krijgslaan 281, 9000 Ghent, Belgium,

18 Thomas.Hermans@ugent.be

\section{Abstract}

In the context of aquifer thermal energy storage, we conducted a hydrogeophysical experiment emulating the functioning of a groundwater heat pump for heat storage into an aquifer. This experiment allowed the assessment of surface electrical resistivity tomography (ERT) ability to monitor the 3D development over time of the aquifer thermally affected zone. The resistivity images were converted into temperature. The images reliability was evaluated using synthetic tests and the temperature estimates were compared to direct temperature measurements. Results showed the capacity of surface ERT to characterize the thermal plume and to reveal the spatial variability of the aquifer hydraulic properties, not captured from borehole measurements. A simulation of the experiment was also performed using a groundwater flow and heat transport model calibrated with a larger set-up. Comparisons of the simulation with measurements highlighted the presence of smaller heterogeneities that strongly influenced the groundwater flow and heat transport. 
32 Keywords: aquifer thermal energy storage, thermally affected zone, monitoring, electrical resistivity

33 tomography, time-lapse, inversion

\section{Introduction}

35 The reduction of fossil fuel consumption is an objective for preserving non-renewable energy resources and reducing the impact of global warming. In this context, renewable and sustainable energies are promoted, e.g. Energy Efficiency Directive 2012/27/EU (European Council, 2012). Smart systems that use heat pumps to transfer heat to or from the ground can take advantage of the thermal stability of the subsurface to reduce energy consumption (Lo Russo et al., 2009; Vanhoudt et al., 2011; Sarbu and Sebarchievici, 2014). Beyond the different existing systems, ground source heat pumps present an inherent thermal resistance with boreholes, while groundwater heat pumps (GWHP) directly use groundwater that presents a relatively stable temperature over seasons. GWHP provide space heating or cooling, domestic hot water production, and are even used to store thermal energy, depending on the season and/or on the specific needs of the infrastructure (Allen and Milenic, 2003; Lo Russo and Civita, 2009). GWHP function through an open loop between two wells or groups of wells drilled in a shallow aquifer. During summer and/or any space cooling periods, water is extracted from the so-called cold well to cool down the infrastructure with the help of heat exchangers (geocooling). The thermal energy in excess, captured by heat exchangers, is then transferred to groundwater before its re-infiltration into the so-called warm well (Gringarten and Sauty, 1975; Ausseur et al., 1982; Voigt and Haefner, 1987). In winter, the GWHP functions in a reverse mode: water is pumped from the warm well and heat is transferred from groundwater to the building. Groundwater is then re-injected into the cold well with a lower temperature (Ausseur et al., 1982; Ampofo et al., 2006). Theoretically, the heat stored in the aquifer during space cooling periods allows an energy reduction for space heating and inversely with the cold stored during space heating periods for space cooling. Such systems are called aquifer thermal energy storage systems (Sommer et al., 2013, 2014; Bridger and Allen, 2014; Possemiers et al., 2015). GWHP working in shallow aquifers requires a relatively small ground surface area by comparison to ground source heat pumps. Thus the thermally affected zone (TAZ) is limited to a volume around boreholes (Sarbu and Sebarchievici, 2014). However, the induced temperature variations in the aquifer likely modify the medium properties such as the chemical composition and water quality (Bonte et al., 2013; Jesußek et al., 2013). Those changes might in turn impact the system efficiency but also the biodiversity, the microbial activities and consequently the ecosystem functions (Griebler et al., 2016). 
64 In addition to controlling the temperature impact often imposed by regulations (Haehnlein et al., 65 2010), the design of GWHP requires a good understanding of the aquifer and heat flow conditions.

66 In particular, issues of thermal short-circuit or recycling between cold and warm wells have to be carefully considered (Banks, 2009; Galgaro and Cultrera, 2013; Milnes and Perrochet, 2013). The propagation of the heat and cold plumes in the aquifer is also highly sensitive to possible variations of hydraulic gradient that could be induced by existing water wells and/or the drilling of additional wells close to the pumping system (Lo Russo et al., 2014) and to the heterogeneity of the subsurface (Bridger and Allen, 2014; Sommer et al., 2013, 2014; Possemiers et al., 2015; Hermans et al., 2018). Methods supplying insights on the heat or cold plume's propagation in the aquifer have to be developed, notably for delimiting the TAZ, to better anticipate the possible difficulties arising from GWHP implementation. Models of groundwater flow and heat transport are often calibrated with empirical values or from local measurements in boreholes (Lo Russo and Civita, 2009; Liang et al., 2011; de Paly et al., 2012; Mattsson et al., 2008; Raymond et al., 2011), ignoring the heterogeneity of the hydrogeological medium. Monitoring the 4D evolution of the TAZ through time is then particularly relevant. The relative proximity of the TAZ to the surface enables a monitoring with noninvasive geophysical methods (Hermans et al., 2014).

Electrical resistivity tomography (ERT) is particularly sensitive to the porous medium temperature (Rein et al., 2004; Revil et al., 1998; Hayley et al. 2007). Moreover, ERT applied in time-lapse (TL) provides spatially distributed information on the changes over time of the porous medium and may target salinity, water content or temperature (for a review on TL ERT see Singha et al., 2015). Thus, TL ERT is specifically appropriate to monitor heat plume development (Hermans et al., 2014). Acquisition systems can work autonomously, allowing the repeated measurements required to achieve sufficient temporal resolution to follow the 3D TAZ development. The method is also minimally invasive and requires low implementation costs compared to a dense network of boreholes. So far, the ability of ERT to monitor heat plumes has been demonstrated in 3D in a laboratory experiment at a scale of a few tens of centimeters (Giordano et al., 2016). Field 2D set-ups confirmed its relevance for monitoring heat storage, tracing experiments and borehole heat exchanger either

92 from surface and/or cross-boreholes measurements (Hermans et al., 2012; Hermans et al., 2015; 93 Giordano et al., 2017; Cultrera et al., 2017). However, 2D interpretation can be limited by out-of-the94 plane or shadow effects during inversion which can limit the quantitative assessment of temperature 95 (Nimmer et al., 2008). This effect is probably partly responsible for discrepancies observed between 
97 use of 3D surveys and subsequent inversion can largely improve imaging of complex 3D subsurface 98 objects (e.g., Van Hoorde et al., 2017).

100 In this paper, we image the development of the TAZ during a heat injection and storage experiment 101 using a 3D ERT survey. The accuracy of ERT-derived temperature estimates is explored through 102 synthetic cases that emphasize the method's sensitivity to target depth and thickness. Results show 103 the ability of surface TL ERT to monitor the 3D development of the TAZ in a shallow aquifer. We 104 compare our results with direct temperature measurements which demonstrates the ability of ERT to 105 supply complementary insights about the sub-surface spatio-temporal dynamic. The ERT-derived temperatures show a general agreement with direct observations, although important discrepancies are observed in the amplitude of the measured variations. The latter are mainly explained by the different representative volumes of the two techniques and the limitations related to the regularized ERT inversion. A groundwater flow and heat transport model calibrated during a previous experimental set-up at a larger scale, is also computed. Its comparison with direct and ERTderived temperature measurements underline the presence of local heterogeneities at the vicinity of the injection well which should be incorporated in the flow and transport model.

\section{Field experiment}

\subsection{Hydrogeological context}

115 The study site is located on the alluvial plain of the Meuse River at Hermalle-sous-Argenteau, $13 \mathrm{~km}$ 116 north-east from Liège, Belgium. From borehole logs analysis, the subsurface medium can be divided 117 into four lithological units. The first layer is composed of loam and presents a thickness of about 1 $118 \mathrm{~m}$. Below, the second unit is constituted of sandy loam, gravels and clay to a depth of $3 \mathrm{~m}$. Between 1193 and $10 \mathrm{~m}$ depth, the third layer is composed of gravel and pebbles in a sandy matrix. This third layer 120 hosts the alluvial aquifer. It can be divided in two main units: the upper aquifer, between 3 and $6 \mathrm{~m}$ 121 depth, composed of sandy gravels and the lower aquifer, between 6 and $10 \mathrm{~m}$ depth, characterized by 122 coarser and cleaner gravels. The water table lies approximately at a depth of $3.2 \mathrm{~m}$. Below $10 \mathrm{~m}$ depth, 123 the basement of the aquifer consists of low permeability carboniferous shale and sandstone (Fig. 1). 
a)
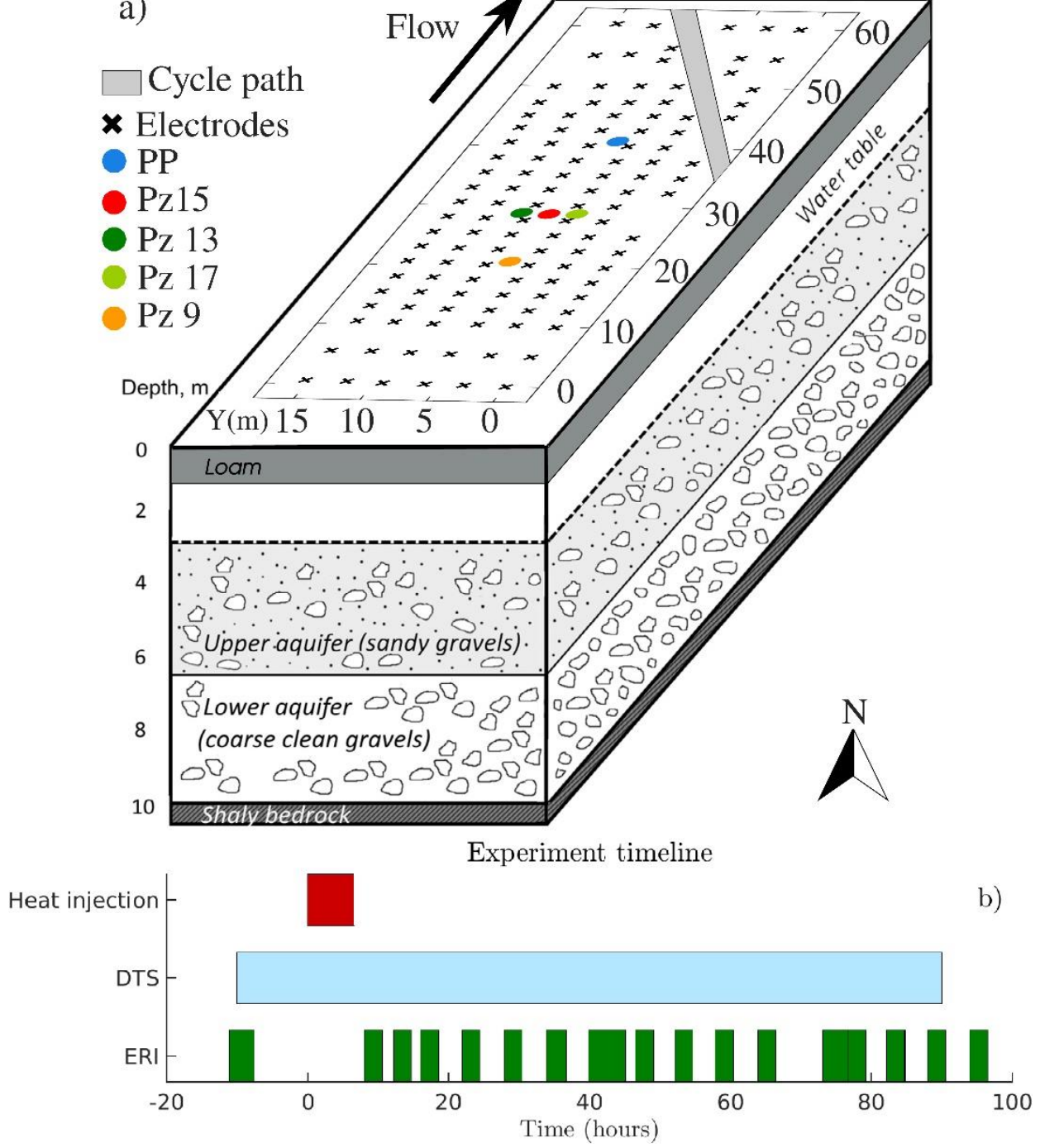

125 Figure 1: a) Scheme of the experimental set-up and structure of the underground medium (modified

126 from Klepikova et al., 2016). b) The experiment timeline shows the duration of the heat injection and 127 the DTS measurements as well as the moment of ERT acquisitions.

129 The site topography is almost flat and the natural hydraulic gradient in the aquifer is approximately $130 \quad 0.06 \%$ with a north-east direction (Brouyère, 2001). Previous experiments showed that the aquifer is 131 characterized by a high average permeability and by a horizontal and vertical heterogeneity 132 (Dassargues, 1997; Derouane and Dassargues, 1998; Brouyère, 2001). In particular, the upper and 133 the lower parts of the aquifer, ranging respectively in [3-6] $\mathrm{m}$ depth and [6-10] $\mathrm{m}$ depth present a 134 respective effective porosity of $4 \%$ and $8 \%$ as estimated from tracer test experiments (Brouyère, 135 2001). Direct measurements of Darcy fluxes were performed to estimate the groundwater flow 136 variations with depth. The lower part of the aquifer present Darcy fluxes in the range of [1-8]x $10^{-3}$ $137 \mathrm{~m} / \mathrm{s}$, about one order of magnitude higher than in the upper layer where values range in [1-10]x 10 ${ }^{-4}$ $138 \mathrm{~m} / \mathrm{s}$ (Wildemeersch et al., 2014). In a previous multiple tracer tests experiment, the monitoring of the 
$1393 \mathrm{D}$ spreading of a heat plume resulting from the injection of heated water in the aquifer also showed

140 lateral variations of the medium hydraulic properties (Klepikova et al., 2016), strengthened by 2D 141 cross-borehole ERT and DTS measurements (Hermans et al., 2015). They confirmed the higher flow

142 in the lower part of the aquifer and the lateral heterogeneity of the aquifer that presents zones of 143 preferential flow (Hermans et al., 2015). Similarly, the thermal properties of the aquifer vary with 144 depth as assessed during the ThermoMap project (Bertermann et al., 2013). The thermal conductivity 145 was estimated at 1.37 and $1.86 \mathrm{~W} / \mathrm{mK}$ and the volumetric heat capacity at 2.22 and $2.34 \mathrm{MJ} / \mathrm{m}^{3} \mathrm{~K}$ in 146 the layers located respectively at depths between 3 to $6 \mathrm{~m}$ and 6 to $10 \mathrm{~m}$.

\section{$147 \quad 2.2$ Heating and injection procedure}

148 Water was pumped from the aquifer at the pumping well PP (Fig. 1a), its initial temperature was $14913.4^{\circ} \mathrm{C}$ and the pumping flow rate was fixed to $3 \mathrm{~m}^{3} / \mathrm{h}$. The water was then heated using a mobile 150 water flow heater (Swingtec AQUAMOBIL DH6 system) before being re-injected with a flow rate 151 of $3 \mathrm{~m} / \mathrm{h}$. The heated water was injected in the borehole $\mathrm{Pz} 15$ between 4.5 and $5.5 \mathrm{~m}$ depth (Fig. 1). $152 \mathrm{Pz} 15$ is located in between two boreholes ( $\mathrm{Pz} 13$ and $\mathrm{Pz} 17$ ) screened on the whole aquifer depth 153 allowing DTS measurements (Fig. 1). The water was heated to a temperature of $42^{\circ} \mathrm{C}$, i.e. with an 154 increase of $28.6^{\circ} \mathrm{C}$ from the initial aquifer temperature. The hot water injection lasted 6 hours, but at 155 the end of the injection step, water was injected at a temperature of $14.5^{\circ} \mathrm{C}$ during 20 minutes due to 156 a technical issue with the water heater. Afterward, a heat storage phase lasted 4 days (Fig. 1b). The 157 injected hot water volume is about $18 \mathrm{~m}^{3}$ that can be used to estimate the order of magnitude of the 158 heat plume development. The thermal plume can be assumed to develop in a $3 \mathrm{~m}$ height cylinder in 159 the upper part of the aquifer that presents a porosity of $4 \%$. Thus, the thermally affected zone should 160 present a maximum volume of $450 \mathrm{~m}^{3}$ (ignoring conduction effects), developing in a cylinder of about 16114 m diameter.

\subsection{D electrical resistivity data acquisition}

163 Electrical resistivity measurements were performed from a grid of electrodes located at the surface to 164 monitor the 3D heat plume evolution in the aquifer. 126 electrodes were placed along 6 profiles of 16521 electrodes centered on the injection well and parallel to the natural flow direction (Fig. 1a). Along 166 each profile the electrodes spacing was $2.5 \mathrm{~m}$ for the 17 central electrodes. Two electrodes at either 167 end of each profile were spaced $5 \mathrm{~m}$ apart. That arrangement of electrodes was selected to allow a 168 finer resolution around the injection well together with a greater penetration depth than profiles with 169 equidistant electrodes. Some electrodes located on the northern side of the profiles 1 to 3 could not 170 be hammered into the ground since the field is crossed by a concrete bike path. Thus, 1 or 2 electrodes 171 were missing on those profiles (Fig. 1a). The electrode profiles were separated by $3 \mathrm{~m}$ so the electrode 
172 grid covers the whole space available to image the plume without degrading the resolution

173 perpendicular to the profiles. Data were acquired with gradient and dipole-dipole protocols along 174 each profile (2D data acquisition). Cross-line measurements were also acquired (Van Hoorde et al., 175 2017), but ignored during inversion due to their low quality. We used an ABEM Terrameter LS 176 connected to a relay switch ES10-64 allowing the acquisition in one shot of the whole data set for the 177 entire electrode grid. A first data acquisition was performed the day before the heated water injection 178 for reconstructing the background image. During the heat storage period 16 acquisitions were 179 performed; once every six hours (Fig. 1b). For the background acquisition, reciprocal measurements 180 were acquired for the whole data set to estimate the measurement error (LaBrecque et al., 1996). 181 Reciprocal measurements correspond to a swap of the electrodes used for current injection and 182 voltage measurements during the "normal" acquisition. A complete normal data set consisted in 3045 183 voltage measurements. For the time-lapse acquisition, the amount of reciprocal data was reduced to 1841119 to speed up the acquisition. The acquisition delay was set to $0.3 \mathrm{~s}$ and the acquisition time was $1850.5 \mathrm{~s}$. The acquisition duration was 2.5 and 1.5 hours for the background and the time-lapse 186 acquisitions, respectively.

\subsection{Borehole measurements}

188 Single-ended optical fibers were inserted in the Pz13 and Pz17 boreholes located on both side of the 189 injection borehole (Fig. 1a). The optical fibers allow a direct monitoring of the temperature variations 190 in the aquifer during the experiment, performed with an AP Sensing Linear Pro Series N4386. The 191 distributed temperature sensing (DTS) measurements provide a spatial sampling of $0.2 \mathrm{~m}$ and a 192 temporal resolution of $2 \mathrm{~min}$. Since the aquifer vertical extension is relatively small (7 $\mathrm{m})$ we choose 193 to preserve the spatial resolution provided by the spatial sampling provided by the DTS. Instead, we 194 preferred reducing the temporal resolution so we applied a running average through time over a 19520 min window. Two sections of the optical fibers were placed respectively in a chilled and a warm 196 water bath. The cold bath was maintained near $0^{\circ} \mathrm{C}$ (about $13^{\circ} \mathrm{C}$ cooler than the aquifer) by regularly 197 adding ice. The baths' temperature was monitored with Pt100 sensors. The cable ends were placed at 198 the bottom of the boreholes due to the small diameter of boreholes compared to the critical bend 199 radius of optical fibers. Thus the differential attenuation of the light along the single-ended cables 200 could not be estimated (Hausner et al., 2011). Nevertheless, the calibration baths allowed to correct 201 the estimated temperatures, guaranteeing their temporal consistency throughout the experiment. DTS 202 measurements were used for checking the relative temperature change $\Delta T$ from the initial state and 203 not for an absolute monitoring of the aquifer temperature. The air temperature increased by $15^{\circ} \mathrm{C}$ at 204 the end of the experiment. However, this effect is attenuated with depth and, although we can observe 
205 a difference at a depth of $1 \mathrm{~m}$ in the DTS measurements, no temperature variation below $3 \mathrm{~m}$ 206 (saturated zone) is visible.

\section{Groundwater flow and heat transport model}

\section{3.1 Settings of the hydrogeological model}

209 We used the 3D groundwater flow and heat transport model HydroGeoSphere (HGS) (Therrien et al.,

210 2010) developed by Klepikova et al. (2016) for the Hermalle-sous-Argenteau experimental site to

211 simulate numerically our experiment and compare temperatures derived from ERT and measured

212 directly with DTS to the simulation. This deterministic model has been constructed and calibrated

213 based on historical data (Dassargues, 1997; Derouane and Dassargues, 1998; Brouyère, 2001) and a

214 multiple tracer experiment, including heat tracer (Wildemeersch et al., 2014; Hermans et al., 2015).

216 The model geometry corresponds to the one described by Klepikova et al. (2016), except that we 217 refined the grid around the injection well to accurately model the experiment. In several aspects their 218 experiment differs from the one presented here. They injected the heat tracer at the base of the Pz 9 219 borehole (Fig. 1a), so in the lower unit of the aquifer that is hydraulically more conductive. 220 Furthermore, $\mathrm{Pz} 9$ is screened over the whole thickness of the aquifer (Table 1) allowing heat 221 propagation upward along the borehole. Their experimental design was adapted to constrain the 222 hydraulic conductivity values on the whole aquifer section. In our experiment the temperature 223 changes occurred in the upper part of the aquifer where heat was injected. Therefore, our data were 224 more sensitive to the spatial variability of hydraulic conductivity distribution in the upper area. 225 Moreover, they injected the heated water at a rate of $3 \mathrm{~m}^{3} / \mathrm{h}$ during 24 hours and 20 minutes, while 226 pumping at a constant discharge rate of $30 \mathrm{~m}^{3} / \mathrm{h}$ in the pumping well (Fig. 1) in order to speed up the 227 heat propagation in the aquifer. Therefore their experiment supplied information on the hydraulic 228 properties in the whole domain in between the injection borehole Pz 9 and the pumping well (Fig1). 229 In our case, we injected and stored the heat in the $\mathrm{Pz} 15$ borehole so our experiment is more sensitive 230 to hydraulic properties distribution in a smaller region surrounding that borehole.

232 Table 1: Main differences between the experiment of Klepikova et al. (2016) and the one presented 233 in this study.

\begin{tabular}{|l|c|c|}
\hline & This study & Klepikova et al., 2016 \\
\hline Depth of injection & $5 \mathrm{~m}$ & $9 \mathrm{~m}$ \\
\hline Duration of injection & 6 hours & 24 hours and 20 minutes \\
\hline
\end{tabular}




\begin{tabular}{|l|c|c|}
\hline Pumping time & Only during injection & All along the experiment \\
Pumping rate & $3 \mathrm{~m}^{3} / \mathrm{h}$ & $30 \mathrm{~m}^{3} / \mathrm{h}$ \\
Injection rate & $3 \mathrm{~m}^{3} / \mathrm{h}$ & $3 \mathrm{~m}^{3} / \mathrm{h}$ \\
\hline Injection well & $\mathrm{Pz} 15$ & $\mathrm{Pz} 9$ \\
\hline Injection well screen interval & $-[5.5 ; 4.5] \mathrm{m}$ & $-[8.7 ; 3.2] \mathrm{m}$ \\
\hline
\end{tabular}

235 The inversion process ran by Klepikova et al. (2016) sought the hydraulic conductivity distribution 236 of the Hermalle site aquifer, in between the Pz 9 and the pumping well PP from a monitoring of the 237 temperature changes in the upper and lower part of the aquifer from 11 observation boreholes. They 238 estimated the hydraulic property values with the pilot point method to parametrize the inversion 239 (Fig. 2). Here we use their resulting model to simulate the heat transport in the aquifer with the 240 characteristics of our experiment. The model includes the density effects due to temperature changes 241 (Graf and Therrien, 2005).

242
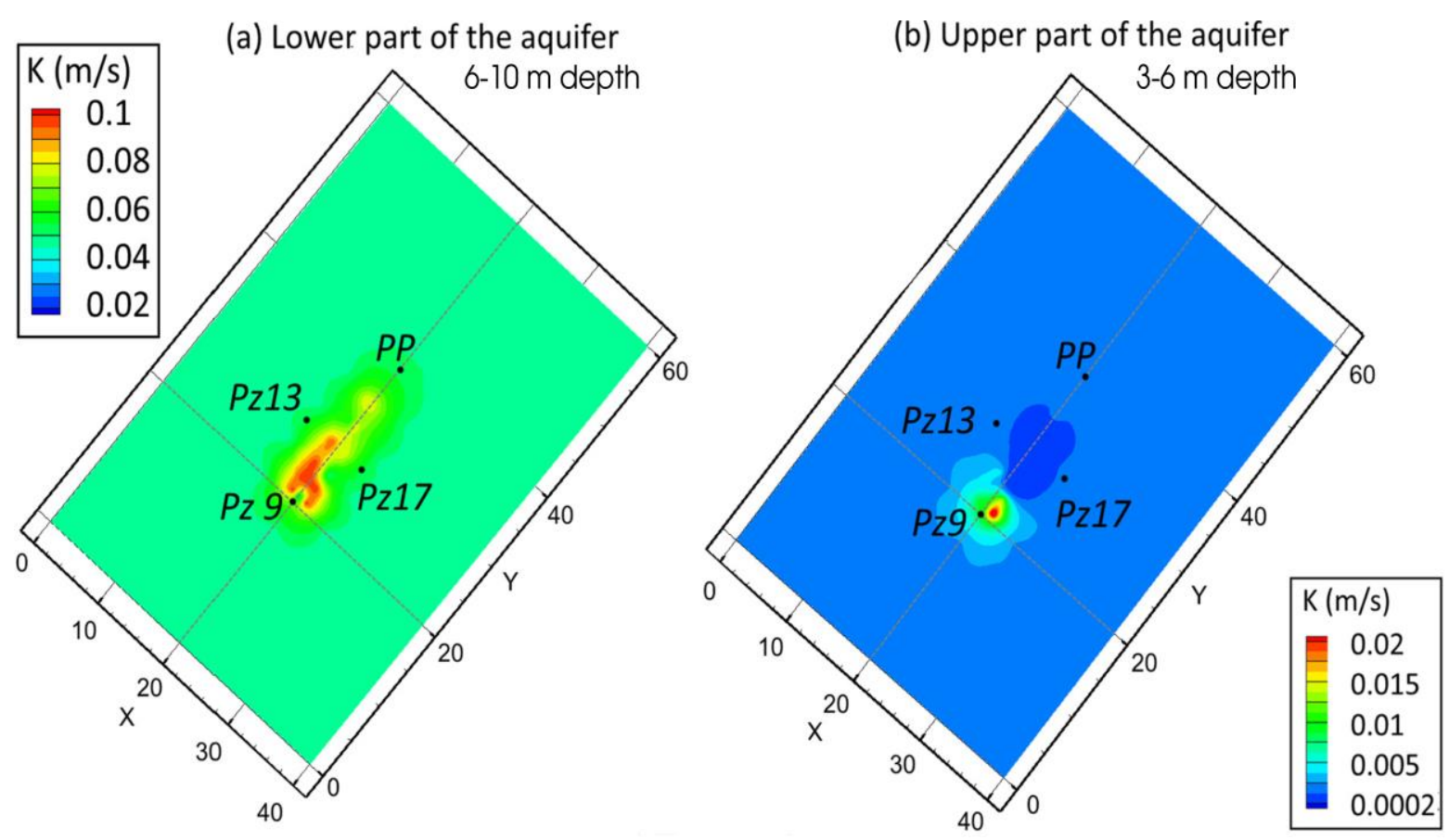

243 Figure 2: Spatial variability of the hydraulic conductivity $\mathrm{K}(\mathrm{m} / \mathrm{s})$ models in XY planes for the lower 244 (a) and upper (b) parts of the aquifer obtained from the inversion of transient temperature responses 245 (Klepikova et al., 2016).

\subsection{Temperature estimates from the HGS model}

247 The temperature variations from the HGS model show a TAZ with a half-sphere geometry in the 248 upper part of the aquifer (Fig. 3). In the lower part of the aquifer, the TAZ presents a tail along the X 249 axis (towards NE which is the main flow direction; Fig. 3 c, d). The higher hydraulic conductivity 
250 and the greater porosity in the lower part of the aquifer (Fig. 2 a) favors heat propagation with 251 groundwater flow through convection. $8 \mathrm{~h}$ after the beginning of the injection, the diameter of the 252 TAZ showing a $\Delta T$ of $4^{\circ} \mathrm{C}$ at a depth of $4.5 \mathrm{~m}$ is about $5 \mathrm{~m}$ and is slightly more elongated in the $\mathrm{X}$ 253 direction. The lower diameter of the model TAZ compared to the above estimate for a cylinder of $25414 \mathrm{~m}$ diameter can be explained by the fact that the model predicts a deeper propagation of the TAZ. 255 Moreover, in the lower part of the aquifer the porosity is set higher in the model than the one used for 256 the cylinder volume evaluation. $47 \mathrm{~h}$ after the injection started, the dimension of the model TAZ 257 showing a $\Delta T$ of $4{ }^{\circ} \mathrm{C}$ is slightly reduced to a diameter of about $3 \mathrm{~m}$ at a depth of $4.5 \mathrm{~m}$. However at 258 that time, $\Delta T$ in the middle of the TAZ is significantly lower, e.g. from $12^{\circ} \mathrm{C}$ after $8 \mathrm{~h}$ (Fig. $3 \mathrm{c}$ ) to $2594{ }^{\circ} \mathrm{C}$ for the same position after $47 \mathrm{~h}$ (Fig. $3 \mathrm{~d}$ ).

260
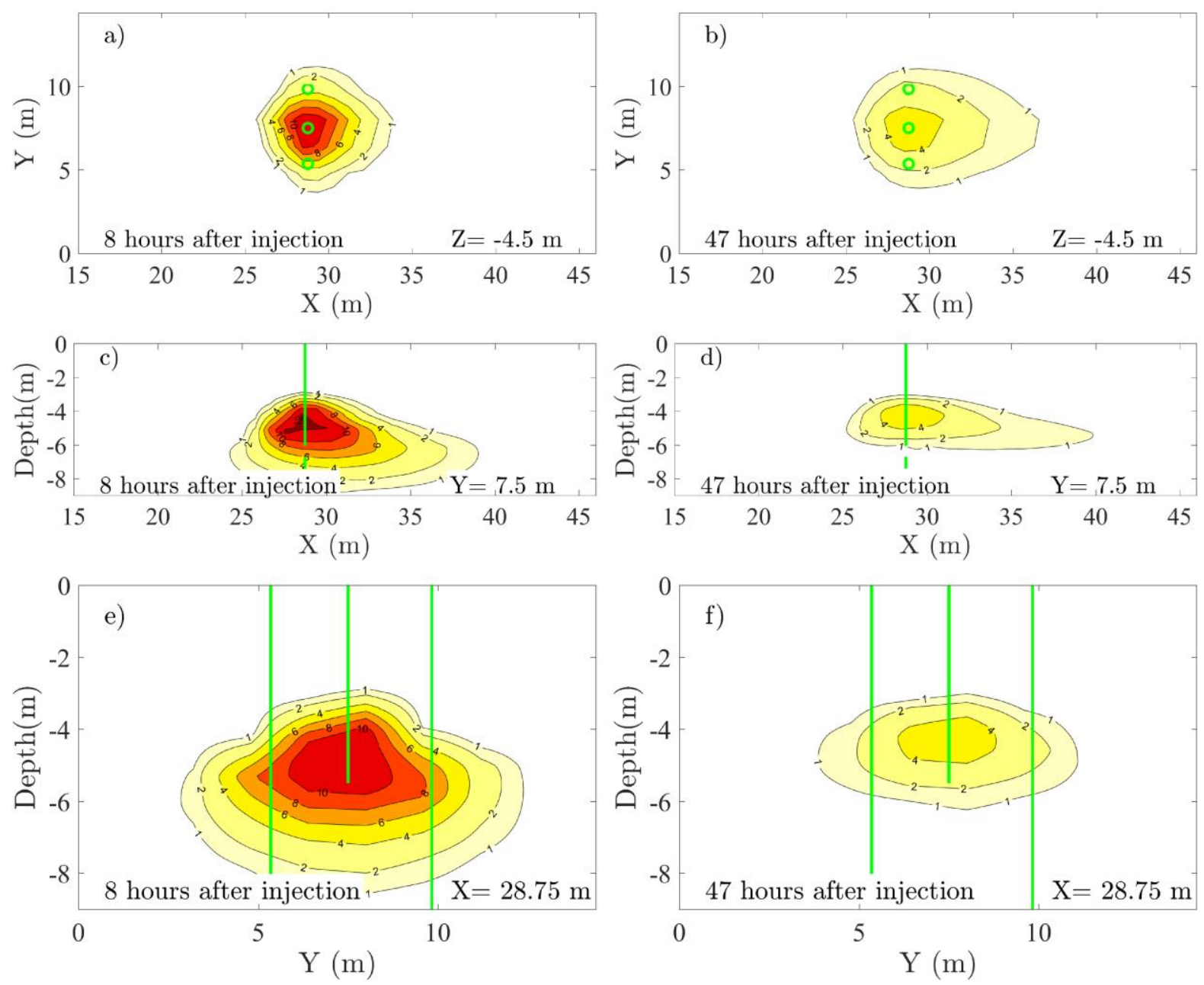

$\Delta \mathrm{T}\left({ }^{\circ} \mathrm{C}\right)$

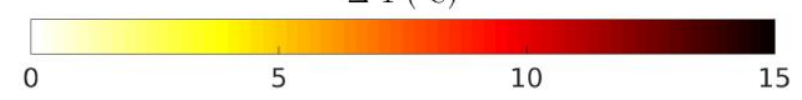

261 Figure 3: Temperature variation from the initial state estimated by the HydroGeoSphere model, $8 \mathrm{~h}$ 262 and $47 \mathrm{~h}$ after the injection started on cross-sections in a horizontal layer at $-4.5 \mathrm{~m}(\mathrm{a}, \mathrm{b})$, on cross263 section at the level of the injection borehole along the electrode profiles $(\mathrm{c}, \mathrm{d})$ and perpendicular to 
264 the electrode profiles (e, f). The green circles and lines represent the injection and measurement

265 boreholes. The black dotted lines and crosses correspond to the electrodes.

\section{6}

267

268

269

270

271

272

274

275

276

277

278

279

280

281

282

283

\section{ERT-derived temperature images reconstruction process}

\subsection{Inversion method}

The image reconstruction process in ERT aims to determine the spatial distribution of bulk resistivity in the medium that best reproduces the resistance measurements. An inverse problem is then solved to iteratively minimize the objective function $\Psi(m)$ :

$$
\Psi(m)=\Psi_{d}(d, m)+\lambda \Psi_{m}(m)
$$

Equation 1

where $\Psi_{d}(d, m)$ measures the data misfit, $\Psi_{m}(m)$ defines model constraints for the inversion regularization and $\lambda$ is the damping factor that balances the weight of the regularization. We used the EIDORS software for solving the inversion of the ERT data (Polydorides and Lionheart, 2002; Adler et al., 2015).

The data misfit $\Psi_{d}(d, m)$ is evaluated between observed measurements $d$ (electrical resistances) and calculated data $f(m)$ computed from a model of resistivity $m$. Model and data are both expressed using their base 10 logarithm.

For the reconstruction of the medium background, before the introduction of perturbation through heat injection, the absolute values of the medium resistivity are sought and the data misfit is expressed as:

$$
\Psi_{d}(d, m)=\sum_{i=1}^{N} \frac{\left|d_{i}-f_{i}(m)\right|^{2}}{\left|\epsilon_{B, i}\right|^{2}}
$$

Equation 2

where $\epsilon_{B}$ are weighting factors accounting for the uncertainty of the measurements.

For time-lapse inversions, the inversion seeks the variations of resistivity from the background model. The measure of the data misfit is here defined using the difference between the background data $d_{0 i}$ (the subscript 0 refers to the background data) and the monitored data $d_{i}$ :

$$
\Psi_{d}(d, m)=\sum_{i=1}^{N} \frac{\left|\left(d_{i}-d_{0 i}\right)-\left(f_{i}(m)-f_{i}\left(m_{0}\right)\right)\right|^{2}}{\left|\epsilon_{T L, i}\right|^{2}}
$$


295 where $m_{0}$ stands for the model parameter distribution of the background state and $\epsilon_{T L}$ corresponds to the time-lapse data weighting.

298 The insertion of prior information on the medium structure is performed by $\Psi_{m}(m)$ in the inversion 299 to stabilize the inverse problem:

$$
\Psi_{m}=|| W_{m}\left(m-m_{0}\right) \|^{2}
$$

302 where $W_{m}$ represents the regularization matrix that is here the identity matrix (Tikhonov, 1963). The background image $m_{0}$ was the result of a first inversion seeking the resistivity values of a medium constituted by four horizontal layers as highlighted by borehole logs analysis and previous ERT experiments (Hermans \& Irving, 2017). The four layers correspond to a superficial unit above $1 \mathrm{~m}$ depth, below the unsaturated zone extends to $3 \mathrm{~m}$ depth, then to a depth of $10 \mathrm{~m}$ lies the aquifer over the bedrock. The depth of the interfaces are taken from borehole logs analysis. In the time-lapse reconstruction scheme, $m_{0}$ corresponds to the background image that refers to the initial state of the medium.

311 The computation of $f(m)$ is performed using a 3D finite element model built using the Netgen 312 software (Schöberl, 1997). The unstructured mesh is refined close to the electrodes for a better 313 accuracy of the resistance estimation, while elements are coarser further away from the electrodes 314 allowing a reduced computation time. A regular coarse mesh was designed for the inversion in order to reduce the number of model parameters and thus stabilize the inversion procedure.

317 The inversion is performed using an iterative Gauss-Newton scheme and at each step a linear search 318 against data misfit seeks the optimum value of the regularization parameter $\lambda$ that minimizes the 319 weighted residuals $\chi$ :

$$
\chi=\sqrt{\frac{1}{N} \Psi_{d}(d, m)} .
$$

322 The inversion is stopped either when the inversion converges, that is when further iteration do not 323 provide a better data fit $\chi$, i.e. no reduction of the objective function $\Psi(m)$ is observed, or when the 324 desired misfit $\chi=|1-\xi|$ is reached, with $\xi=10^{-2}$. Such a stopping criteria avoids over-fitting data 
and the presence of artefacts in the resulting image (Kemna, 2000). The data analysis and the method used to determine the error models weighting the data misfit are described in the appendix A.1.

\subsection{ERT conversion to temperature images}

Temperature variations in the aquifer induce a change of the medium electrical properties, i.e. the resistivity and its inverse the conductivity. The conductivity variations from the initial state and the temperature changes are related by a linear petrophysical relationship so temperature images can be derived from ERT (Hermans et al., 2014). We consider that bulk conductivity variations in the aquifer are due to changes of the fluid conductivity that depends on fluid salinity and temperature variations. We checked that chemical perturbations produced by temperature changes have a negligible effect on the fluid salinity (Hermans et al., 2015). Thus, the conductivity increase can be quantitatively interpreted in terms of temperature change using the linear relationship:

$$
\frac{\sigma_{f, T}}{\sigma_{f, 25}}=m_{f}(T-25)+1
$$

where $\sigma_{f, T}$ stands for the fluid conductivity at a temperature $T$ and $m_{f}$ corresponds to the fractional change of the fluid conductivity per degree Celsius around the reference temperature $T=25^{\circ} \mathrm{C}$. From water samples taken on site the trend between the temperature and the fluid conductivity was estimated to be $m_{f}=0.0194$ and the fluid conductivity at $T=25^{\circ} \mathrm{C}$ was evaluated at $\sigma_{f, 25}=$ $0.0791 \mathrm{~S} / \mathrm{m}$ (Hermans et al., 2015).

Temperature change from the initial state $\Delta T$ images can be constructed from the observed variations of the bulk conductivity $\sigma_{b}$ by converting them with (Hermans et al., 2014; 2015):

$$
\Delta T=\frac{1}{m_{f}}\left(\frac{\sigma_{b, T L}}{\sigma_{b, B}} \frac{\sigma_{f, B}}{\sigma_{f, 25}}-1\right)+25-T_{\text {init }}
$$

$\sigma_{b, B}$ and $\sigma_{b, T L}$ correspond respectively to the bulk conductivity of the background state and of a timelapse acquisition. $\sigma_{f, B}=0.0614 \mathrm{~S} / \mathrm{m}$ represents the fluid conductivity at the initial state and was estimated from Eq. (6) with an initial temperature of $T_{\text {init }}=13.44^{\circ} \mathrm{C}$. That initial temperature value corresponds to the average of the temperatures measured along both boreholes using DTS before the heat injection. The estimate of the initial conductivity of the fluid was validated with a value of $\sigma_{f, B}=$ $0.0598 \mathrm{~S} / \mathrm{m}$ by direct measurement with a CTD probe in the injection borehole. 
355 We ran synthetic simulations computed from models of a cylindrical plume with different thicknesses and positions in the aquifer in order to evaluate the sensitivity of the electrode array to the target depth and thickness. The temperature increase in the cylinder was fixed to $17^{\circ} \mathrm{C}$ and the cylinder diameter to $8 \mathrm{~m}$ based on the outcomes of the HGS model. The cylinder was centered on the injection borehole Pz 15 (Fig. 1). The tested cylinder heights varied between $1.5 \mathrm{~m}$ and $5 \mathrm{~m}$. The cylinder upper limit positions checked was of $-3 \mathrm{~m}$ and $-4 \mathrm{~m}$ so it is always located below the unsaturated zone (Fig. 4). On all images, we observe that the inversion allows a fairly correct reconstruction of the target shape when the target is located close enough to the electrodes (approximately the distance corresponding to the electrode spacing) or sufficiently thick to be detectable (at least the electrode spacing). We note

364 that the target upper limit is always overestimated by about $2 \mathrm{~m}$ and that the amplitude of $\Delta T$ decreases with depth. The region of higher $\Delta T$ in the reconstructed target is also shifted upward the center of the actual target. Moreover, the temperature increase leaks out of the synthetic target delimitation, except in the shallow region where artifacts induce a reduction of the electrical conductivity (Fig. 4). Quantitatively we can compare the estimated temperature increase at the level of the injection borehole Pz15 to the synthetic target as summarized in Table 2. We note that when the target is relatively shallow, that is for an upper limit at $-3 \mathrm{~m}$, the increase of the target thickness greatly helps determining more closely the target true temperature. However if the target is only $1 \mathrm{~m}$ deeper, the target thickness increase does not allow accurate estimates of the target temperature.

Table 2: Percentage of the temperature increase estimated by ERT from the synthetic target increase for different cylinder height and upper position.

\begin{tabular}{|c|c|c|c|c|}
$\begin{array}{c}\text { Height } \backslash \\
\text { Upper limit }\end{array}$ & $1.5 \mathrm{~m}$ & $2.5 \mathrm{~m}$ & $4 \mathrm{~m}$ & $5 \mathrm{~m}$ \\
\hline$-3 \mathrm{~m}$ & $24 \%$ & $44 \%$ & $67 \%$ & $78 \%$ \\
\hline$-4 \mathrm{~m}$ & $17 \%$ & $30 \%$ & $41 \%$ & $46 \%$ \\
\hline
\end{tabular}

The target blur and the global temperature underestimation are related to the regularization that favors a smooth change from the background model. The smoothing effect hinders a precise location of the target and thus an overestimation of the target depth. Moreover, the estimation of $\Delta T$ at depth is degraded by the reduction of the ERT sensitivity with depth (Fig. 4). From those tests, we infer that the geometry of the plume (its size and depth) is fairly estimated, with an overestimation of the plume vertical position when the target depth and thickness correspond to the electrode inter-distances.

383 Furthermore, images underestimate the actual temperature changes in the aquifer. Smoothing effects 
and the underestimation of the aquifer parameters using TL-ERT are well known and results from the regularization procedure in the inversion (Singha and Moysey, 2006).

386
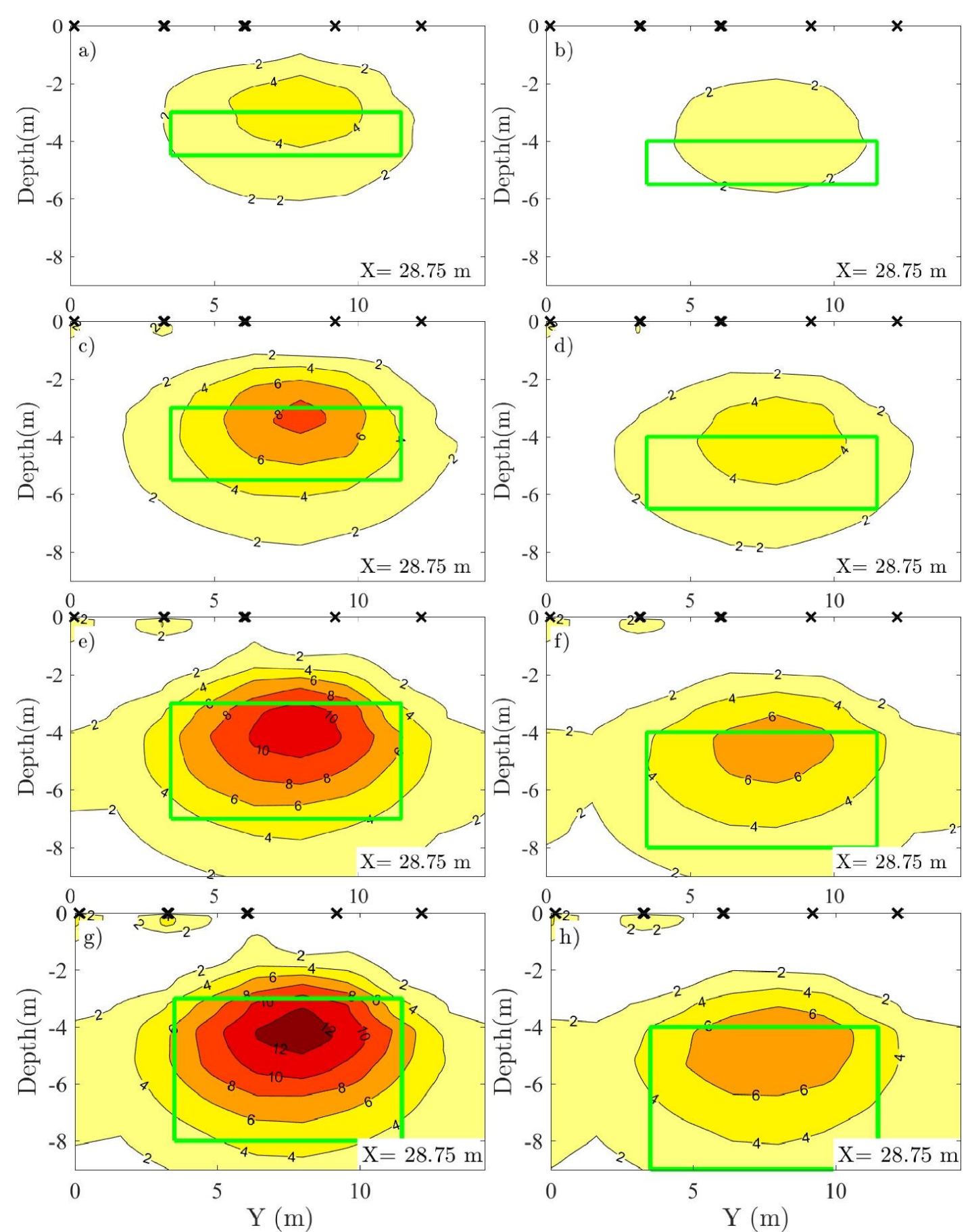

$\Delta \mathrm{T}\left({ }^{\circ} \mathrm{C}\right)$

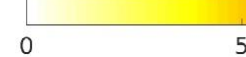

10

387 Figure 4: Temperature variation estimates from synthetics built as a vertical cylindrical heat plume.

388 The cylinder thickness is of $1.5(\mathrm{a}, \mathrm{b}), 2.5(\mathrm{c}, \mathrm{d}), 4(\mathrm{e}, \mathrm{f})$ and $5 \mathrm{~m}(\mathrm{~g}, \mathrm{~h})$ and its upper limit is at $-3 \mathrm{~m}$ 389 (a, c, e, g) and $-4 \mathrm{~m}(\mathrm{~b}, \mathrm{~d}, \mathrm{f}, \mathrm{h})$. The green lines indicate the shape of the cylindrical target, the black 390 crosses correspond to the electrodes. 


\subsection{Background images}

393 The 3D inversion of the background ERT data set provides the distribution of the subsurface 394 resistivity with a root mean square of the data misfit of $1.03 \%$. The results are presented by $2 \mathrm{D}$ vertical 395 cross-sections corresponding to each acquisition profile (Fig. 5). The images show the vertical 396 variations of the electrical resistivity corresponding to the four lithological units. The superficial 397 conductive layer with a thickness of $1 \mathrm{~m}$ and a resistivity of about $115 \Omega . \mathrm{m}$ corresponds to the loam 398 unit. Between 1 and $3 \mathrm{~m}$ deep the medium presents heterogeneous variations of resistivity with zones 399 of a few meters length showing a resistivity higher than $400 \Omega$.m and locally values reaching $4001000 \Omega . m$ while the surrounding medium presents a resistivity of $250 \Omega . m$. Here the resistive regions 401 might be interpreted by lenses of clean unsaturated gravels in a medium of sandy loam and gravels.

402 Below, in the aquifer layer at a depth between 3 and $10 \mathrm{~m}$ the medium presents a median resistivity 403 of $180 \Omega$.m with some more conductive areas notably below profiles located on $\mathrm{Y}=6$ and $9 \mathrm{~m}$ where 404 the resistivity is around $100 \Omega$.m. Those conductive anomalies are likely an effect of the pumping 405 well metallic-casing. We note that we do not distinguish any clear resistivity variations related to the 406 different nature of the aquifer upper and lower units. Previous ERT experiments discriminated 407 between the upper and lower regions through a higher resolution cross-borehole survey (Hermans et 408 al., 2015), or through a specific analysis developed for discriminating the different facies of the 409 medium (Hermans and Irving, 2017), which were not used in this study. Finally, the lower layer 410 located below $10 \mathrm{~m}$ depth in the bedrock shows a median resistivity of $280 \Omega . \mathrm{m}$. Those values are in 411 accordance with previous ERT investigations on the site (Hermans and Irving, 2017). 


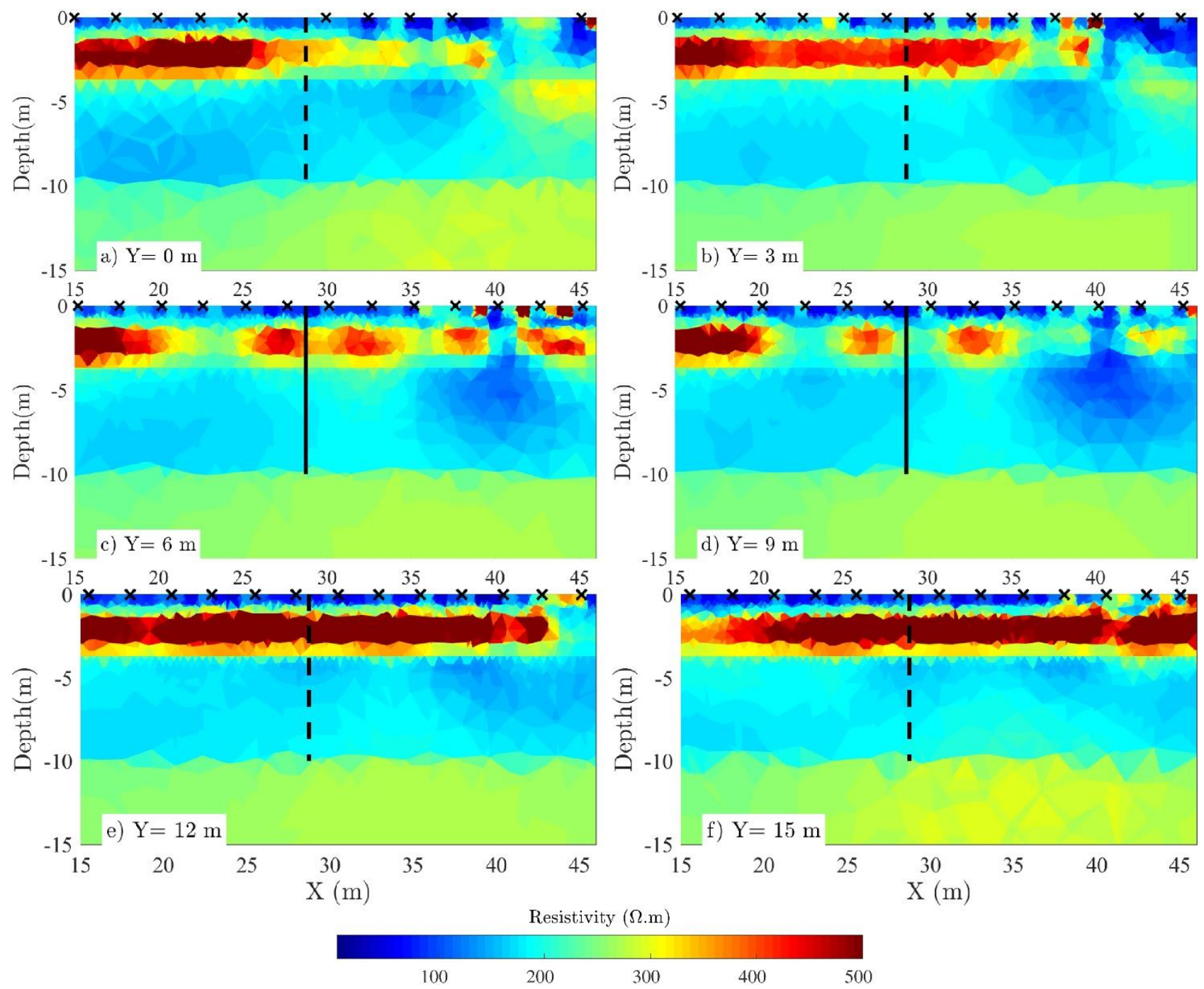

412 Figure 5: Electrical resistivity cross-sections extracted below each electrode profile from the 3D 413 model. The black hashed or solid lines represent the injection and measurement boreholes projection.

414 The black crosses correspond to the electrodes.

415

\section{$416 \quad 5.2$ Time-lapse conductivity variation images}

417 Since conductivity variations vary linearly with temperature we choose to represent that property 418 instead of resistivity variations over selected time steps ( $8 \mathrm{~h}$ and $47 \mathrm{~h}$ after the injection started) on 419 sections of the 3D model (Fig. 6). A conductive plume, with a variation between 10 and $30 \%$ from 420 the initial state, is observed in the upper part of the aquifer (Fig. 6c to f). The region showing a 421 conductivity increase higher than $10 \%$ presents an oval geometry with a horizontal extent of about $4225 \mathrm{~m}$ along the $\mathrm{X}$ axis and $10 \mathrm{~m}$ in the $\mathrm{Y}$ direction (Fig. $6 \mathrm{a}$ and $\mathrm{b}$ ). The plume is not centered on the 423 injection borehole, but is slightly shifted along the $\mathrm{X}$ axis, in the same direction as the natural 424 groundwater flow. The region with the highest conductivity change is also slightly shifted in the Y 425 direction. The global shape of the zone affected by conductivity changes does not significantly evolve with time, but the conductivity further increases more than 50 hours after the end of the injection. A 
second conductive target is observed at $\mathrm{Y}=0 \mathrm{~m}$, below the first profile which is lacking central

428 electrodes. We interpret this more conductive zone as an artifact related to a lower sensitivity in that 429 region due to the missing electrodes (Fig. 6). The images also present regions with a conductivity 430 decrease from the initial state, mostly in the shallow region but also at depth below profile 1 and at 431 the extremity of the central profiles. In the shallow region, they might be related to changes in the 432 medium saturation above the aquifer. Below, we interpret them as artifacts that are strengthened by 433 our less conservative choice of error model (see Appendix A.1).

434
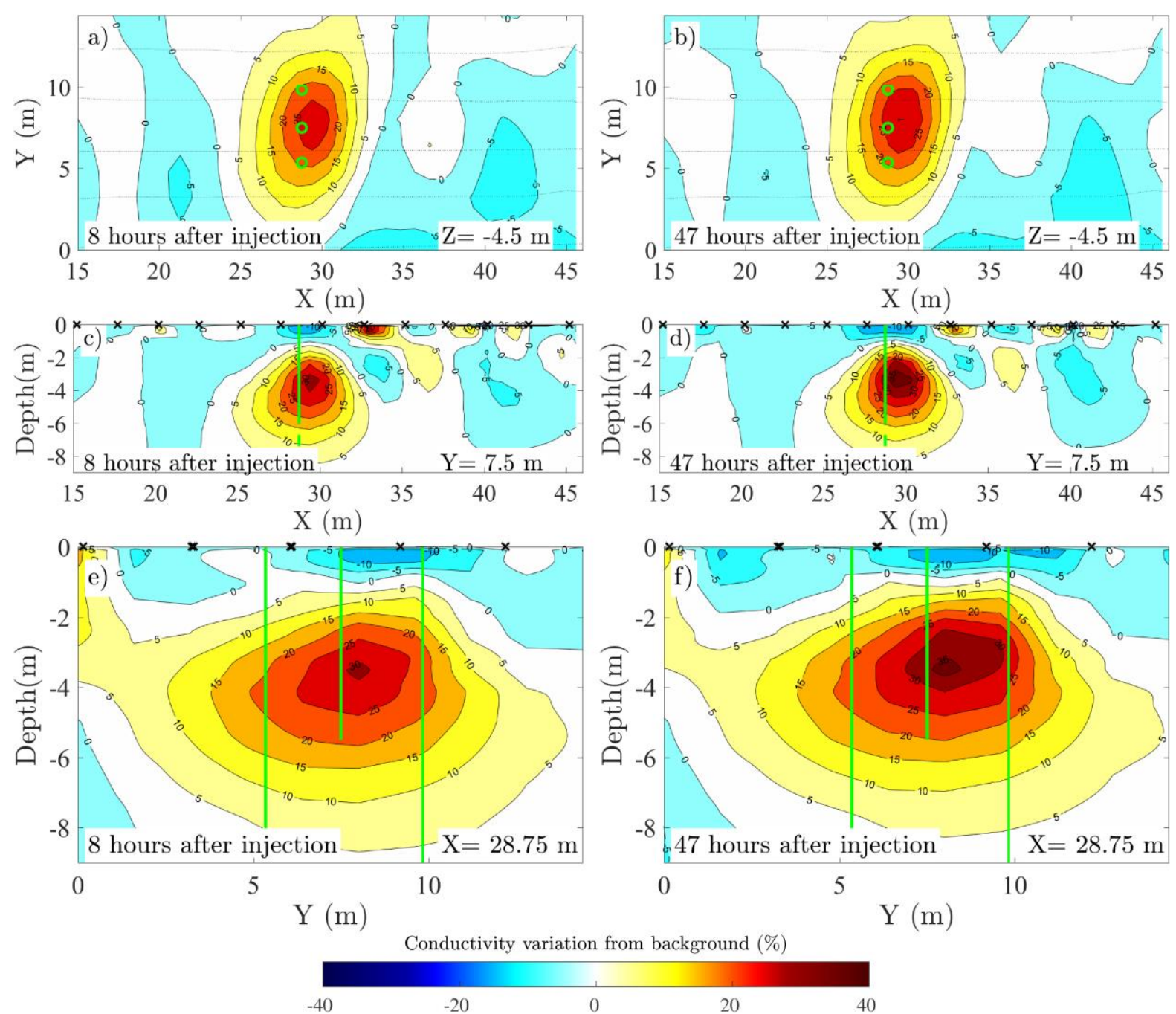

435 Figure 6: Conductivity variations $8 \mathrm{~h}$ and $47 \mathrm{~h}$ after the injection started with respect to the 436 background measurements in a horizontal layer at $-4.5 \mathrm{~m}(\mathrm{a}, \mathrm{b})$, on cross-section at the level of the 437 injection borehole along the electrode profiles (c, d) and perpendicular to the electrode profiles (e, f). 438 The green circles and lines represent the injection and measurement boreholes. The black dotted lines 439 and crosses correspond to the electrodes. 
441 ERT-derived $\Delta T$ images show a similar shape and evolution of the TAZ as the conductivity variation 442 images (Fig. 7). On those images, $\Delta T$ is fixed to $0^{\circ} \mathrm{C}$ in regions showing negative contrasts of 443 conductivity since we interpret them as artifacts. The TAZ with a $\Delta T$ increase of $4^{\circ} \mathrm{C}$ from the initial 444 state presents an oval shape in the horizontal plane at $\mathrm{Z}=-4.5 \mathrm{~m}$ with a length of $10 \mathrm{~m}$ in the $\mathrm{Y}$ 445 direction and a width of $5 \mathrm{~m}$ along the $\mathrm{X}$ axis (Fig. 7a, b). As on the conductivity variation images, 446 the shift along the $\mathrm{X}$ and $\mathrm{Y}$ directions of the TAZ with a highest $\Delta T$ is observed (Fig. 7c, d, e, f). 447 From the synthetic tests, we deduce that the TAZ oval shape is not an effect of the electrode design 448 or inversion, but reflects the actual shape of the TAZ. This highlights the usefulness of ERT in 449 providing information on the plume anisotropy. ERT-derived $\Delta T$ images show a relatively shallow 450 TAZ upper limit, in the region where the method sensitivity is still adequate for detection. However, 451 as observed from the synthetic inversions, the TAZ upper limit deduced from ERT might be 452 overestimated by $2 \mathrm{~m}$. The TAZ should indeed be confined below the water table ( $3 \mathrm{~m}$ depth, Fig. 1). 453 Similarly to the synthetic cases, it is difficult to properly image the shape of the TAZ in the lower 454 part of the aquifer from ERT inversions, due to the low sensitivity (Fig. 4). Quantitatively, 8 hours 455 after the beginning of injection, the derived $\Delta T$ reaches locally $12^{\circ} \mathrm{C}$ in the upper part of the aquifer 456 between the injection borehole and the electrode profile at $\mathrm{Y}=9 \mathrm{~m}$ (Fig. 7). 40 hours later, that region 457 shows a higher $\Delta T$ value of $14^{\circ} \mathrm{C}$. However, a difference of only $2^{\circ} \mathrm{C}$ is in the range of uncertainty 458 observed in the synthetic tests, it is thus difficult to confirm this $2^{\circ} \mathrm{C}$ increase. Nevertheless, we note 459 the persistence of a TAZ on the ERT-derived $\Delta T$ images with a similar shape $8 \mathrm{~h}$ and $47 \mathrm{~h}$ after the 460 injection (Fig. 7). 

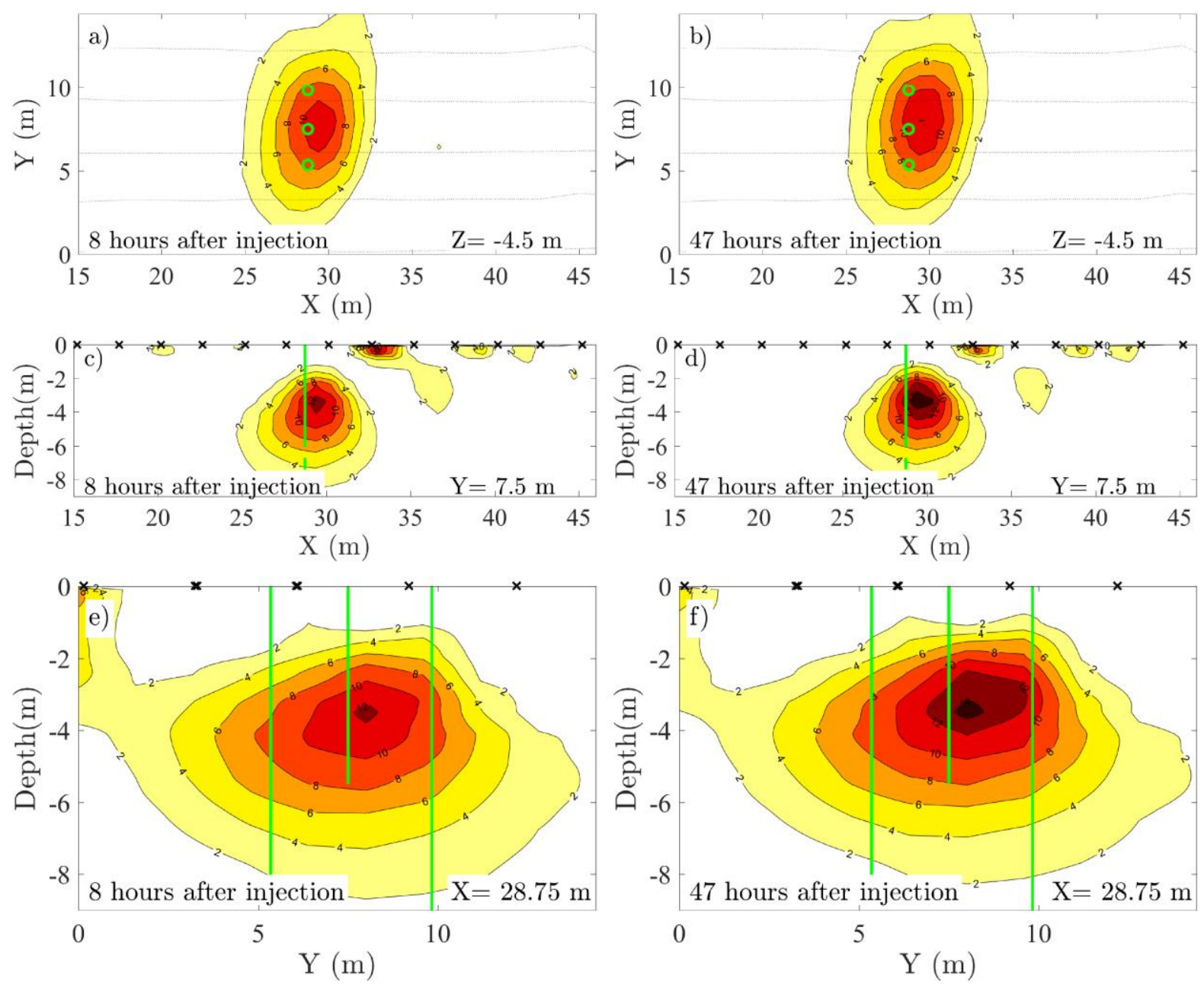

$\Delta \mathrm{T}\left({ }^{\circ} \mathrm{C}\right)$

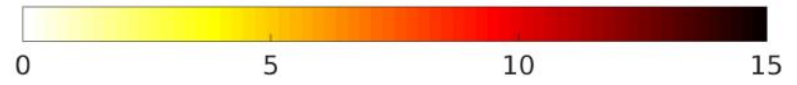

462 Figure 7: Temperature variation from the initial state estimated from ERT $8 \mathrm{~h}$ and $47 \mathrm{~h}$ after the 463 injection started in a horizontal layer at $-4.5 \mathrm{~m}(\mathrm{a}, \mathrm{b})$, on cross-section at the level of the injection 464 borehole along the electrode profiles (c, d) and perpendicular to the electrode profiles (e, f). The green 465 circles and lines represent the injection and measurement boreholes. The black dotted lines and 466 crosses correspond to the electrodes.

468 Predictions from the HGS model and ERT-derived $\Delta T$ results are represented on cross-sections to ease their comparison (Fig. 8). The edges correspond to the TAZ showing a $\Delta T$ of $4^{\circ} \mathrm{C}$ from the initial state, at different times after the injection. Estimates derived from ERT show a coarser TAZ with a shape preserved all along the experiment, probably due to the smoothing effect of the inversion as

472 demonstrated by the synthetic tests. They illustrate the gradual plume shift along the $\mathrm{X}$ and $\mathrm{Y}$ 473 directions. Predictions from the HGS model show a smaller TAZ confined at the level of the hot water 474 injection. The dimension of the predicted TAZ shrinks progressively and vanishes after $58 \mathrm{~h}$. 475 Compared to ERT-derived $\Delta T$ contours, the HGS predictions show also a stronger influence of the 
natural groundwater flow that transports the plume in the $\mathrm{X}$ direction, notably in the lower part of the aquifer. Note that the natural flow was estimated from a regional model (Brouyère, 2001) and has a

478 larger influence in our experiment than in the one used for calibration given the lower pumping rate 479 (Table 1). Finally, the HGS predicted plumes do not show the significant anisotropy with an 480 elongation in the $\mathrm{Y}$ direction in the horizontal plane observed on the ERT results.
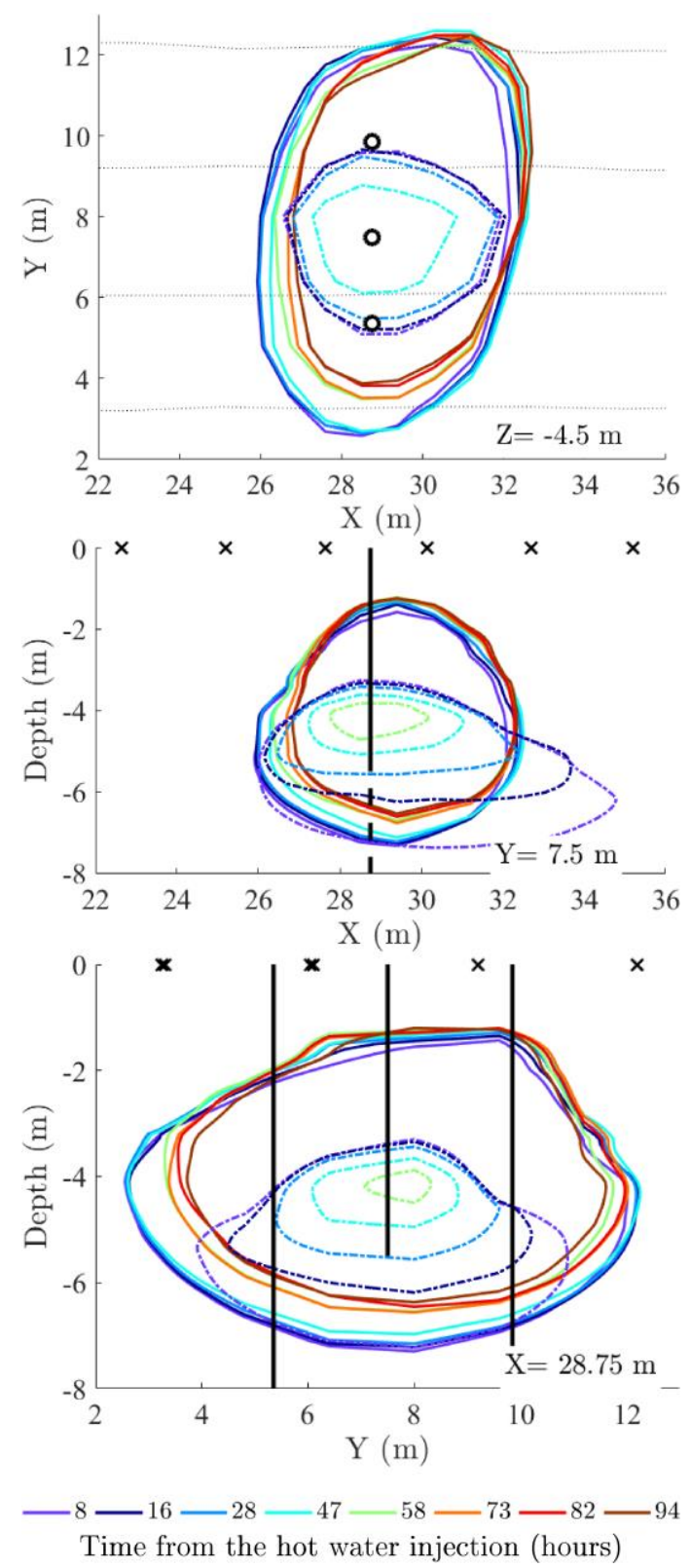

481 Figure 8: Evolution of the plume shape over time, with a contour line at $\Delta T=4^{\circ} \mathrm{C}$. The colored lines 482 represent estimates from ERT and the hashed colored lines predictions from the HGS model. Data 483 are presented on cross-sections in a horizontal layer at $-4.5 \mathrm{~m}$ (a), at the level of the injection borehole 484 along the electrode profiles (b) and perpendicular to the electrode profiles (c). The black circles (a) 485 and lines (b,c) represent the injection and measurement boreholes. The black dotted lines (a) and 486 crosses $(\mathrm{b}, \mathrm{c})$ correspond to the electrodes. 


\subsection{Direct temperature measurements}

488 DTS direct temperature measurements in the aquifer show that the temperature variation from the 489 initial state $\Delta T$ reaches $20^{\circ} \mathrm{C}$ in the upper part of the aquifer, while in the lower part $\Delta T$ is about $10^{\circ} \mathrm{C}$ 490 with a local increase to $15^{\circ} \mathrm{C}$ (Fig. 9). From the end of the injection, $\Delta T$ decreases abruptly at depth 491 below $5 \mathrm{~m}$, while in the upper part $\Delta T$ decreases gradually with time. We also note that measurements 492 performed at $\mathrm{Y}=9.85 \mathrm{~m}$ show a larger and stronger print of the TAZ than measurements performed 493 at $\mathrm{Y}=5.35 \mathrm{~m}$. Thus DTS measurements confirm the plume shift in the $\mathrm{Y}$ direction observed from 494 ERT. During the injection, the heat plume propagates at depth until the bottom of the boreholes 495 (Fig. 9). However, the heat increase in depth might be related to local convection around boreholes 496 and not due to heat propagation through the whole aquifer (Hermans et al., 2015; Klepikova et al., 497 2016).
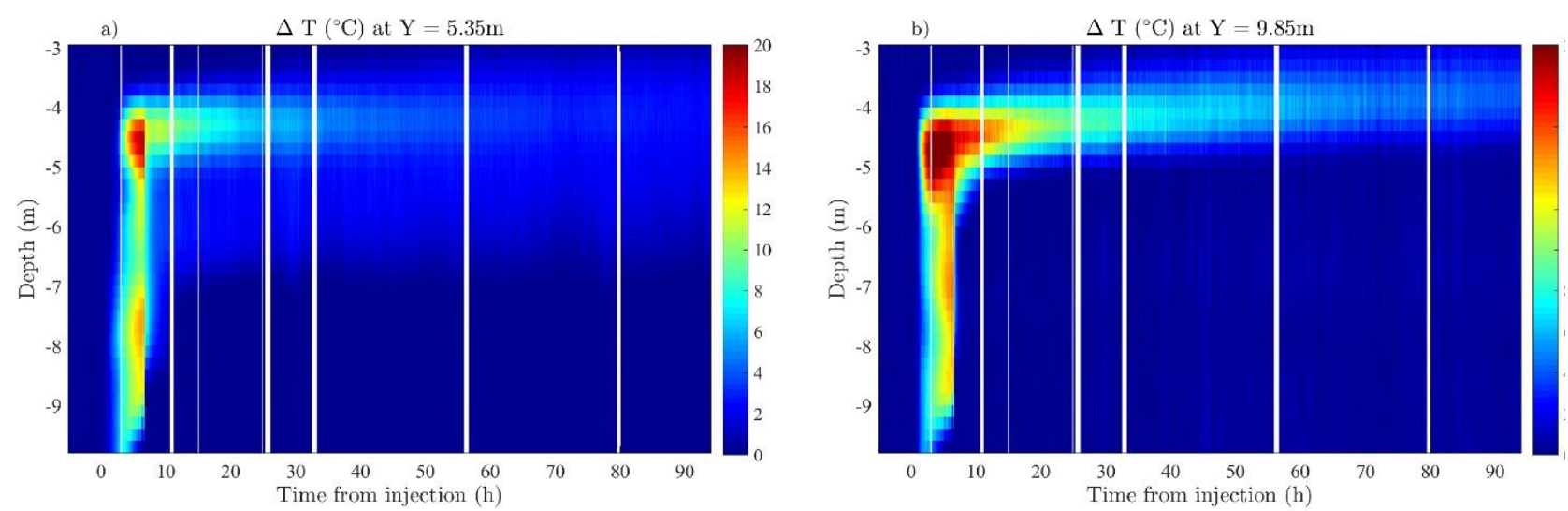

498 Figure 9: Temperature variation from the initial state estimated with the DTS all along the aquifer section at the measurement boreholes Pz17 (a) and Pz13 (b).

\subsection{Comparison of temperature dynamics}

501 The dynamics of $\Delta T$ from direct measurements, ERT estimates and the HGS predictions are compared 502 at the level of the measurement and injection boreholes (Pz 13, 15, 17 Fig. 1) at a depth of $4.5 \mathrm{~m}$ (Fig. 503 10). That depth corresponds to the highest temperature measured with DTS (Fig. 9). Direct $\Delta T$ 504 measurements are acquired using DTS data for Pz 13 and $\mathrm{Pz} 17$ and a CTD probe for the injection 505 borehole Pz 15 (Fig. 1). ERT-derived $\Delta T$ estimates correspond to values extracted from the voxels of 506 the 3D models obtained by the inversion of each data sets acquired after the hot water injection. 507 Similarly, predictions correspond to values extracted from the HGS model voxels at different 508 simulation times. 

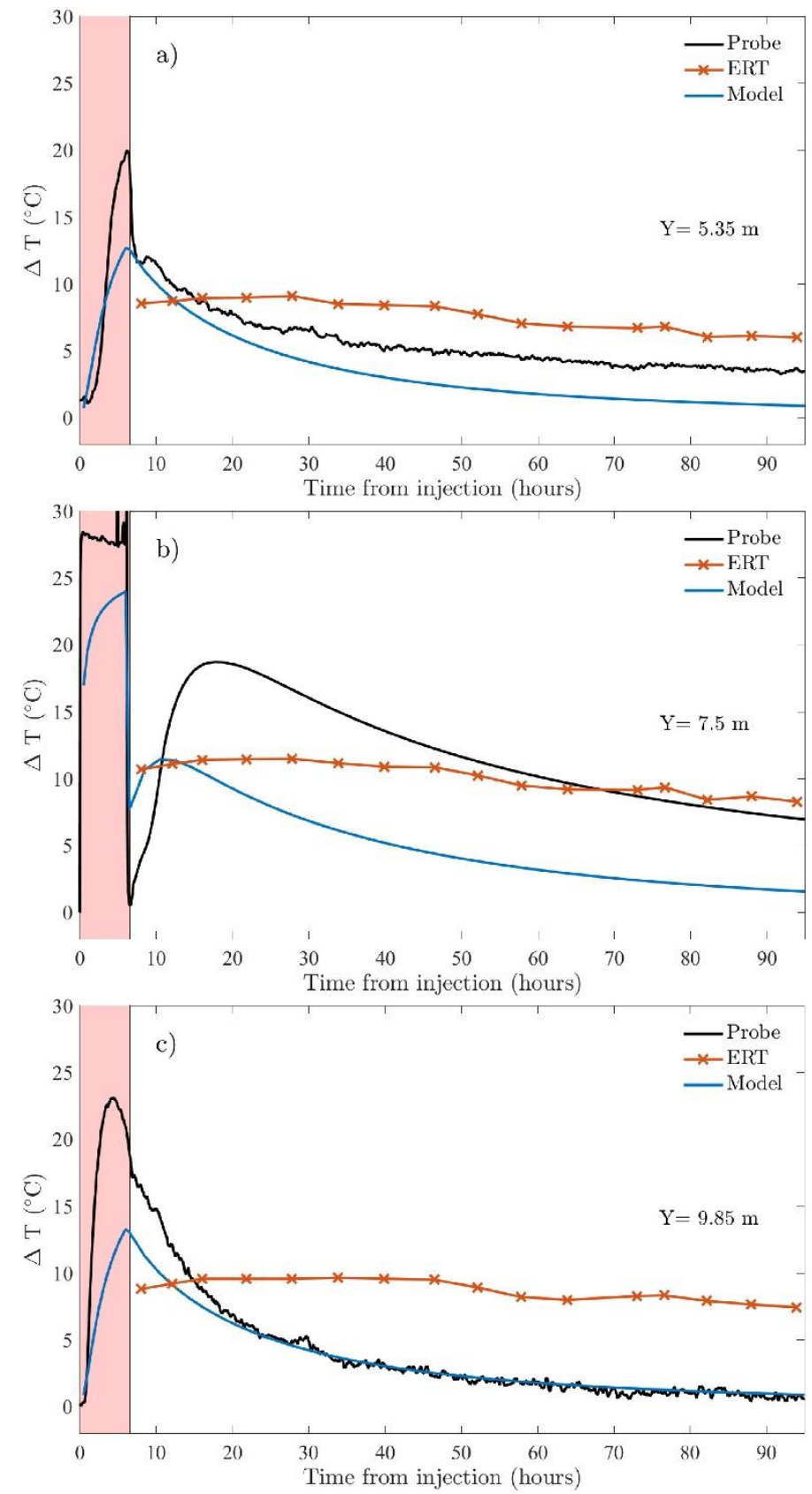

510 Figure 10: Temperature variations from the initial state at a depth of $4.5 \mathrm{~m}$ at the level of $\mathrm{Y}=5.35 \mathrm{~m}$

511 (a), $Y=7.5 \mathrm{~m}$ (injection borehole), b) and $\mathrm{Y}=9.85 \mathrm{~m}$ (c). The red zone represents the duration of the

512 heat injection. ERT estimates are obtained from the 3D time-lapse inversions. Model predictions were

513 computed with the HGS model.

515 The probe located in the injection well ( $\mathrm{Y}=7.5 \mathrm{~m}$, Fig. 10b) shows an average $\Delta T$ value of $27^{\circ} \mathrm{C}$

516 during the injection phase. Then $\Delta T$ decreases sharply to $0.5^{\circ} \mathrm{C}$ and softly increases to a peak value 517 of $19^{\circ} \mathrm{C}$ due to the injection of cold water. $\Delta T$ decreases then gradually to a value of $7^{\circ} \mathrm{C}$ at the end of 518 the experiment. Those direct measurements confirm the persistence of the TAZ all along the 519 experiment as observed with ERT (Fig. 7, 8, 10). DTS measurements in neighboring wells do not 520 show the same rebound pattern but instead a faster $\Delta T$ decrease at the end of the injection phase. In 
521 the measurement borehole at $\mathrm{Y}=5.35 \mathrm{~m}$, direct $\Delta T$ measurements reach $20^{\circ} \mathrm{C}$ during the injection 522 phase and decrease to $3.5^{\circ} \mathrm{C}$ at the end of the experiment (Fig. 10a). The second measurement 523 borehole at $\mathrm{Y}=9.85 \mathrm{~m}$ shows a different dynamic with a higher $\Delta T$ value that reaches $23^{\circ} \mathrm{C}$ during 524 the injection phase but decreases to a lower value of $0.5^{\circ} \mathrm{C}$ at the end of the experiment (Fig. 10c).

ERT-derived $\Delta T$ clearly miss to capture the dynamics of the temperature decrease within the TAZ. $\Delta T$ values are underestimated a few hours after the injection phase and overestimated later (Fig. 10). In general, all $\Delta T$ curves derived from ERT are rather stable, although showing a slight decreasing trend, compared to the direct $\Delta T$ measurements. In particular ERT-derived $\Delta T$ remains higher at the end of the experiment $\left(\Delta T\right.$ of $6^{\circ} \mathrm{C}$ and $7.5^{\circ} \mathrm{C}$ in borehole at $\mathrm{Y}=5.35 \mathrm{~m}$ and $\mathrm{Y}=9.85 \mathrm{~m}$ respectively, Fig. 10). Those observations confirm the results of the synthetic tests, showing that the survey design is mostly sensitive to the global temperature change around the well.

The comparison of the HGS model prediction with the $\Delta T$ direct measurements shows that the model globally underestimates $\Delta T$ values compared to direct measurements (Fig. 10). The HGS model predicts lower $\Delta T$ values at the end of the injection phase in the three boreholes of $12.5^{\circ} \mathrm{C}, 24^{\circ} \mathrm{C}$ and $13.5^{\circ} \mathrm{C}$ for a $\mathrm{Y}$ position of 5.35, 7.5 and $9.85 \mathrm{~m}$ respectively (Fig. 10a). At the end of the experiment the HGS model predicts a $\Delta T$ value of $1.5^{\circ} \mathrm{C}$ in the injection borehole, confirming its inability to represent the TAZ persistence. At that time, in both measurement boreholes show similar $\Delta T$ values of about $1^{\circ} \mathrm{C}$.

\section{Discussion}

542 The experiment performed here demonstrates the capacity of ERT to deliver useful information about 543 the 4D evolution of a thermal plume in a shallow aquifer. ERT-derived $\Delta T$ images supply information 544 on the plume anisotropy in a horizontal plane and its persistence all along the experiment (Fig. 7, 8, 545 10). The plume anisotropy is attested with synthetic tests but cannot be deduced from direct 546 measurements as boreholes close to the injection well are lacking along the $\mathrm{X}$ axis. However, direct 547 measurements in the injection well confirm the TAZ persistence through time (Fig. 10b). So the 548 performed experiment provides an interesting insight on the aquifer storage heat capacity not 549 expected by the HGS model. The revealed anisotropy in the XY direction by ERT might indicate the 550 presence of a preferential water flow path bypassing hydraulic barriers. Such a preferential flow path 551 in the upper part of the aquifer can be related to clay lenses channeling groundwater flow in the $\mathrm{Y}$ 552 direction. Despite TL ERT supplying smoothed results due to regularization, the method has the 553 capacity to provide useful insights about local hydraulic conductivity heterogeneity. The suspected 
554 local heterogeneities have a strong influence on the groundwater flow and hence on heat transport.

555 However, those small heterogeneities are not taken into account in the existing HGS model. The

556 hydraulic parameters used for the simulation should be adjusted around the injection well to 557 reproduce the plume evolution. Indeed, the hydraulic parameters were determined during a previous 558 experiment representative of a larger scale. The integration of hydraulic parameter variations at that 559 smaller scale into the model could help reproducing the horizontal anisotropy of the TAZ 560 development revealed by ERT.

In this specific experiment, although ERT identifies the trend, it misses to accurately identify the temporal fluctuations of $\Delta T$. Indeed, DTS measurements show a significant $\Delta T$ decrease in both monitored boreholes, while ERT only indicates a slight decreasing trend (Fig. 10). The agreement is better in the injection well. Such examples illustrate the difficulty in comparing data representative of $\Delta T$ changes in different volumes. On the one hand, heat loss towards the atmosphere might have favored the $\Delta T$ decrease observed with DTS within wells. On the other hand, since ERT acquisitions are sensitive to temperature changes in a large volume, their estimates might better reflect the matrix heat storage and release. DTS and CTD measurements are bathed in the borehole water and thus not directly connected to the conditions in the aquifer. In addition, the injection of unwanted cold water further complicated the distribution of temperature in the aquifer, creating an anomaly below the resolution of ERT.

Quantitatively, the ability of ERT to image the dynamics compared to direct measurements is also limited since we note an underestimation of ERT-derived $\Delta T$ values a few hours after the injection phase and an overestimation later. This is a direct consequence of the regularization, as highlighted by the synthetic inversions (Singha and Moysey, 2006). In practice, a small plume with high temperature yields the same image as a large plume with a lower temperature. Combined with the slow dynamic characteristic of a storage experiment, it generates ERT images with limited amplitude variations, but with the correct trend.

Due to regularization, ERT-derived $\Delta T$ images also present a shift in the vertical position of the plume and a coarse shape of the TAZ, both identified with synthetic tests. The effect of blur could be attenuated by reducing the acquisition time, by acquiring a limited amount of reciprocal data (Rucker, 2014). Other regularization method such as covariance constraints or minimum-gradient support might help overcome the smoothing effect and improve the delineation of the TAZ (Hermans et al., 2016b; Fiandaca et al., 2015; Nguyen et al., 2016). Furthermore, ERT lacks sensitivity in the 
588

589

590

591

592

593

594

595

596

597

598

599

600

601

602

603

604

605

606

607

608

609

610

611

612

613

614

\section{Conclusion}

616 Although a quantitative estimation of temperature was not possible in this experiment, due to the

617 inherent limitations of inversion, our study demonstrates the pertinence of the minimally invasive 4D

618 ERT method to provide qualitative, complementary insights on the development of a thermal plume

619 in a shallow aquifer. The ERT-derived $\Delta T$ images obtained here inform about the anisotropic shape

620 of the thermal plume and its persistence all along the experiment. Hence, the method indicates the 
621 presence of local heterogeneities at the vicinity of the injection well and the aquifer heat storage 622 capacity confirmed by direct measurements. The anisotropic behavior cannot be validated using direct 623 temperature measurements as it would have required a denser network of boreholes close to the

624 injection well (Fig. 1). Thus, ERT measurements provide crucial information for the set-up of 625 groundwater heat pumps (GWHP), which requires an accurate knowledge of the spatial variability of 626 the aquifer's hydraulic and heat capacity properties. So, the insertion of a correct distribution of the 627 hydraulic properties in the hydrogeological model has to be performed for estimating the likely shape 628 and extension of the TAZ for different GWHP injection regimes. Surface ERT could be used as a 629 control tool for monitoring the successful functioning of GWHP in operation.

631 However, the sensitive analysis we performed show that the ERT sensitivity to the plume decreases 632 strongly with the plume depth and depends also on its dimension. Thus, the qualitative 633 characterization of the TAZ performed through ERT inversion is strongly influenced by the TAZ 634 depth. Indeed, the method struggles to provide the shape of the TAZ in the lower part of the aquifer. 635 In our case, most of the TAZ is really shallow, reaching the groundwater table $3 \mathrm{~m}$ deep, so the 636 method is sensitive to changes induced in the subsurface by the TAZ development. Nevertheless, for 637 a deeper TAZ, the electrode design should be adapted to explore the medium at greater depths. This 638 can be done by using a larger distance between electrodes but at the cost of a lower spatial resolution.

640 The work by Nolwenn Lesparre was supported by the project SUITE4D from a BEcome a WAlloon 641 REsearcher fellowship fund co-financed by the Department of Research Programs of the Federation 642 Wallonia - Brussels and the COFUND program of the European Union. The field experiments was 643 made possible thanks to the F.R.S.-FNRS research credit 4D Thermography, grant number J.0045.16. 644 We gratefully acknowledge Thomas Kremer and Solomon Ehosioke who participated to the field 645 works and data acquisition supervision.

\section{Appendix A.1 Data analysis and error model}

647 We assume that the data (resistance measurements) are uncertain due to noise and that the latter is 648 composed of a random component and a systematic one correlated over time. The effect of systematic 649 error, including modeling errors, can be canceled out by subtraction when working with data650 differences inversion (LaBrecque and Yang, 2001). The weighting factors $\epsilon_{B}$ and $\epsilon_{T L}$ for the 651 background and time-lapse inversions have to be correctly evaluated. As suggested by Slater et al. 652 (2000) and Lesparre et al. (2017), we use the analysis of normal to reciprocal disparities. First, 
653

654

655

656

657

658

659

660

661

662 with

664

665

666

667

668

669

670

671

672

673

resistance data were sorted to remove outliers and the data were selected so they presented a repetitive error lower than $1 \%$ and a reciprocal error lower than $5 \%$ for each time step. The total number of filtered data at each time step was reduced from 3045 to 1948 measurements.

For the background image, an error model $\epsilon_{B}$ was fit to the measured difference $\left|R_{N}-R_{R}\right|$ between the normal $R_{N}$ and reciprocal resistance $R_{R}$ after removing outliers and bining by $\langle R\rangle$. The error $\epsilon_{B}$ varies linearly with resistance (Fig. A.1 a, Slater et al., 2000):

$$
\epsilon_{B}=a_{B}+b_{B}\langle R\rangle
$$

Equation A.1

$$
\left\langle R_{i}\right\rangle=\frac{\left(R_{N, i}+R_{R, i}\right)}{2} \text { for all } i \text { where } \frac{\left|R_{N, i}-R_{R, i}\right|}{\left\langle R_{i}\right\rangle}<0.05
$$

Equation A.2

For the estimate of an error model of the time lapse inversions, normal and reciprocal data at a given time were compared to the background measurements (Lesparre et al., 2017). The normal difference between times $t_{0}$ and $t_{i}$ as $\Delta \log R_{N}=\log R_{N, i}-\log R_{N, 0}$, and the reciprocal difference as $\Delta \log R_{R}=$ $\log R_{R, i}-\log R_{R, 0}$ were used in the time lapse inversions because the data fit were in the logarithmic domain. The difference error model $\epsilon_{T L}$ was then fit to the measured value of the normal-reciprocal discrepancy $\left|\Delta \log R_{N}-\Delta \log R_{R}\right|$ that varies linearly with the inverse of the resistance (Fig. A.1 b, Lesparre et al., 2017):

$$
\epsilon_{T L}=a_{T L}+\frac{b_{T L}}{\langle R\rangle}
$$

Equation A.3
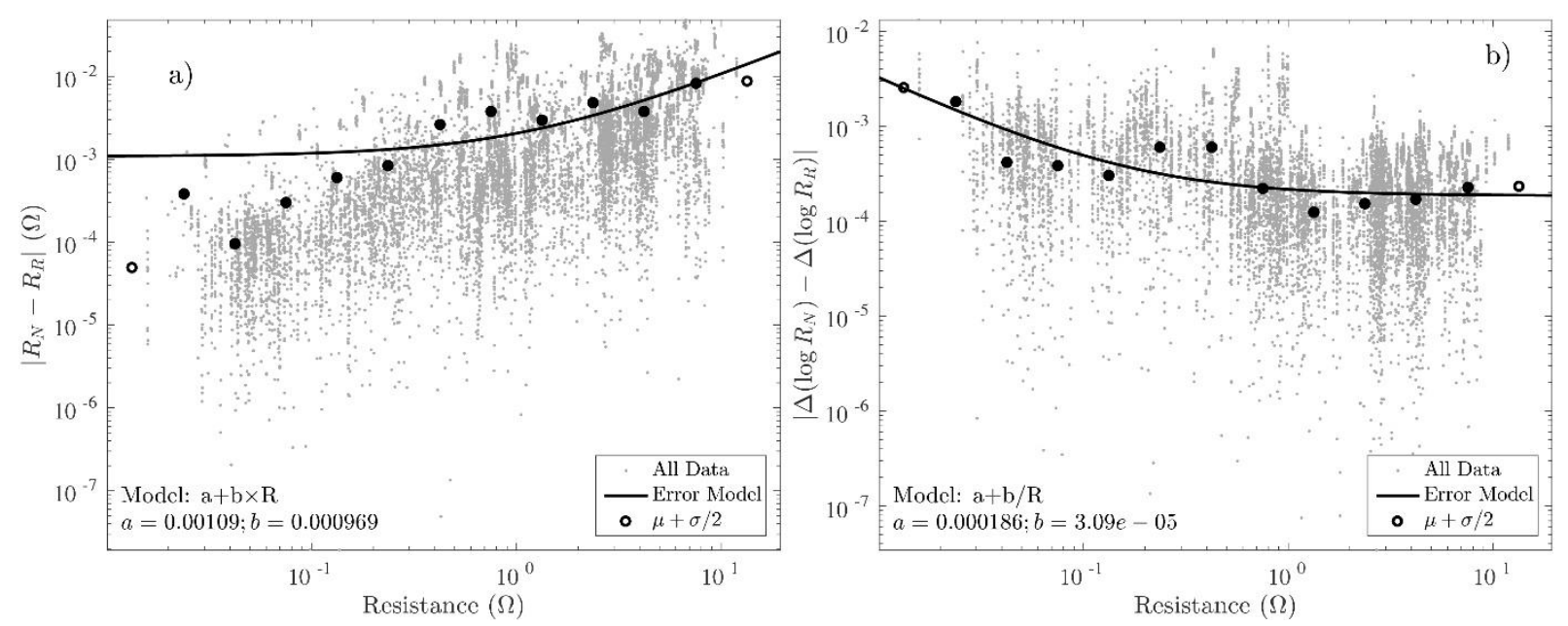

674 Figure A.1: Measured and model error from the normal and reciprocal acquisition for the background

675 (a) and the time-lapse (b) inversions. 
677 The error model parameters $a_{B}, b_{B}, a_{T L}$ and $b_{T L}$ were estimated by fitting the measured errors as a 678 function of $\langle R\rangle$ (Fig. A.1). For the time-lapse error estimate, $\langle R\rangle$ also expresses as stated in Eq. A.2. 679 Error data were divided into classes of $\langle R\rangle$ with four bins per decade of $\bar{R}_{i}$, logarithmically equally 680 spaced. For each bin the average $\mu$ and the standard deviation $\sigma$ were estimated (Koestel et al., 2008). 681 The choice of the error threshold on which the error model is fitted impacts the data weighting and 682 so the residuals $\chi$ that are used as a stopping criteria (see Eq. 5). We choose to fit both error models 683 to $\mu+\sigma / 2$ (Fig. A.1) in order to be more sensitive to conductivity variations due to the hot water 684 injection.

\section{References}

686 Adler, A., Boyle, A., Crabb, M.G., Gagnon, H., Grychtol, B., Lesparre, N., Lionheart, W.R., 2015. 687 EIDORS Version 3.8. In Proc. of the 16th Int. Conf. on Biomedical Applications of Electrical 688 Impedance Tomography.

689 Allen, A., Milenic, D., 2003. Low-enthalpy geothermal energy resources from groundwater in 690 fluvioglacial gravels of buried valleys. Appl. Energy 74, 9-19, http://dx.doi.org/10.1016/S0306$6912619(02) 00126-5$.

692 Ampofo, F., Maidment, G.G., Missenden, J.F., 2006. Review of groundwater cooling systems in 693 London. Appl. Therm. 2055-2062, 694 http://dx.doi.org/10.1016/j.applthermaleng.2006.02.013.

695 Ausseur, J.Y., Menjoz, A., Sauty, J.P., 1982. Stockage couplé de calories et de frigories en aquifère 696 par doublet de forages. J. Hydrol., 56(3), 175-200, http://dx.doi.org/10.1016/0022-1694(82)90012-9.

697 Banks, D., 2009. Thermogeological assessment of open-loop well-doublet schemes: a review and 698 synthesis of analytical approaches. Hydrogeol. J., 17(5), 1149-1155, 699 http://dx.doi.org/10.1007/s10040-008-0427-6.

700 Bertermann, D., Bialas, C., Rohn, J., 2013. ThermoMap - area mapping of superficial geothermic 701 resources by soil and groundwater data. Available at http://geoweb2.sbg.ac.at/thermomap/.

702 Bonte, M., van Breukelen, B.M., Stuyfzand, P.J., 2013. Temperature-induced impacts on 703 groundwater quality and arsenic mobility in anoxic aquifer sediments used for both drinking water 
704 and shallow geothermal energy production. Water Res., 47(14), 5088-5100, 705 http://dx.doi.org/10.1016/j.watres.2013.05.049.

706 Bridger, D.W., Allen, D.M., 2014. Influence of geologic layering on heat transport and storage in an 707 aquifer thermal energy storage system. Hydrogeol. J., 22(1), 233-250, 708 http://dx.doi.org/10.1007/s10040-013-1049-1.

709 Brouyère, S., (Ph.D. thesis), 2001. Etude et modélisation du transport et du piégeage des solutés en 710 milieu souterrain sariablement saturé. University of Liege (unpublished).

711 Cultrera, M., Boaga, J., Di Sipio, E., Dalla Santa, G., De Seta, M., \& Galgaro, A., 2017. Modelling 712 an induced thermal plume with data from electrical resistivity tomography and distributed 713 temperature sensing: a case study in northeast Italy, Hydrogeol. J., 1-15. 714 http://dx.doi.org/10.1007/s10040-017-1700-3

715 Caterina, D., Beaujean, J., Robert, T., Nguyen, F., 2013. A comparison study of different image 716 appraisal tools for electrical resistivity tomography. Near Surf. Geophys., 11(6), 639-657, 717 http://dx.doi.org/10.3997/1873-0604.2013022.

718 Dassargues, A., 1997. Modeling baseflow from an alluvial aquifer using hydraulic-conductivity data 719 obtained from a derived relation with apparent electrical resistivity. Hydrogeol. J., 5(3), 97-108, 720 http://dx.doi.org/:10.1007/s100400050125.

721 Derouane, J., Dassargues, A., 1998. Delineation of groundwater protection zones based on tracer tests 722 and transport modeling in alluvial sediments. Environ. Geol., 36(1-2), 27-36, 723 http://dx.doi.org/10.1007/s002540050317.

724 de Paly, M., Hecht-Méndez, J., Beck, M., Blum, P., Zell, A., Bayer, P., 2012. Optimization of energy 725 extraction for closed shallow geothermal systems using linear programming. Geothermics 43, 57-65, 726 http://dx.doi.org/10.1016/j.geothermics.2012.03.001.

727 European Council, 2012. Directive 2012/27/EU of the European Parliament and of the Council of 25 728 October 2012 on energy efficiency, amending Directives 2009/125/EC and 2010/30/EU and repealing 729 Directives 2004/8/EC and 2006/32/EC. Official Journal of the European Union, Legislative acts. 730 Brussels, Belgium. 
731 Fiandaca, G., Doetsch, J., Vignoli, G., \& Auken, E., 2015. Generalized focusing of time-lapse 732 changes with applications to direct current and time-domain induced polarization inversions. 733 Geophys. J. Int., 203(2), 1101-1112.

734 Galgaro, A., Cultrera, M., 2013. Thermal short circuit on groundwater heat pump. Appl. Therm. Eng., 735 57(1), 107-115, http://dx.doi.org/10.1016/j.applthermaleng.2013.03.011.

736 Giordano, N., Comina, C., Mandrone, G., 2016. Laboratory scale geophysical measurements aimed 737 at monitoring the thermal affected zone in Underground Thermal Energy Storage (UTES) 738 applications. Geothermics, 61, 121-134, http://dx.doi.org/10.1016/j.geothermics.2016.01.011.

739 Giordano, N., Arato, A., Comina, C., Mandrone, G., 2017. Time-lapse electrical resistivity imaging 740 of the thermally affected zone of a Borehole Thermal Energy Storage system near Torino (Northern 741 Italy). J. Appl. Geophys., 140, 123-134, http://dx.doi.org/10.1016/j.jappgeo.2017.03.015.

742 Graf, T., Therrien, R., 2005. Variable-density groundwater flow and solute transport in porous media 743 containing nonuniform discrete fractures. Adv. water resour., 28(12), 1351-1367, 744 http://dx.doi.org/10.1016/j.advwatres.2005.04.011.

745 Griebler, C., Brielmann, H., Haberer, C. M., Kaschuba, S., Kellermann, C., Stumpp, C., Hegler F., 746 Kuntz, D., Walker-Hertkorn, S., Lueders, T., 2016. Potential impacts of geothermal energy use and 747 storage of heat on groundwater quality, biodiversity, and ecosystem processes. Environ. Earth Sci., 748 75(20), 1391, http://dx.doi.org/10.1007/s12665-016-6207-z.

749 Gringarten, A.C., Sauty, J.P., 1975. A theoretical study of heat extraction from aquifers with uniform 750 regional flow. J. Geophys. Res., 80(35), 4956-4962, http://dx.doi.org/10.1029/jb080i035p04956.

751 Haehnlein S., Bayer P., Blum P., 2010. International legal status of the use of shallow geothermal 752 energy. Renew. Sust. Energ. Rev., 14(9), 2611-2625, http://dx.doi.org/10.1016/j.rser.2010.07.069.

753 Hausner, M.B., Suárez, F., Glander, K.E., Giesen, N.V.D., Selker, J.S., Tyler, S.W., 2011. Calibrating 754 single-ended fiber-optic Raman spectra distributed temperature sensing data. Sensors, 11(11), 755 http://dx.doi.org/10859-10879 10.3390/s111110859.

756 Hayley, K., Bentley, L.R., Gharibi, M., Nightingale, M., 2007. Low temperature dependence of 757 electrical resistivity: Implications for near surface geophysical monitoring. Geophys. Res. Lett., 758 34(18), http://dx.doi.org/10.1029/2007gl031124. 
759 Hermans, T., Vandenbohede, A., Lebbe, L., Nguyen, F., 2012. A shallow geothermal experiment in 760 a sandy aquifer monitored using electric resistivity tomography. Geophysics 77(1), B11-B21, 761 http://dx.doi.org/10.1190/GEO2011-0199.1.

762 Hermans, T., Nguyen, F., Robert, T., Revil, A., 2014. Geophysical methods for monitoring 763 temperature changes in shallow low enthalpy geothermal systems. Energies, 7(8), 5083-5118, 764 http://dx.doi.org/10.3390/en7085083.

765 Hermans T., Wildemeersch S., Jamin P., Orban P., Brouyère S., Dassargues A., Nguyen F., 2015. 766 Quantitative temperature monitoring of a heat tracing experiment using cross-borehole ERT. 767 Geothermics, 53, 14-26, http://dx.doi.org/10.1016/j.geothermics.2014.03.013.

768 Hermans, T., Oware, E., Caers, J., 2016a. Direct prediction of spatially and temporally varying physical properties from time-lapse electrical resistance data. Water Resour. Res., 52(9), 7262-7283, 770 http://dx.doi.org/10.1002/2016WR019126.

771 Hermans, T., Kemna, A., Nguyen, F., 2016b. Covariance-constrained difference inversion of time772 lapse electrical resistivity tomography data. Geophysics, 81(5), E311-E322, 773 http://dx.doi.org/10.1190/GEO2015-0491.1.

774 Hermans, T., Irving, J. 2017. Facies discrimination with electrical resistivity tomography using a 775 probabilistic methodology: effect of sensitivity and regularisation. Near Surface Geophysics, 15, 1325, http://dx.doi.org/10.3997/1873-0604.2016047.

Hermans, T., Nguyen, F., Klepikova, M., Dassargues, A., Caers, J. 2018. Uncertainty quantification of medium-term heat storage from short-term geophysical experiments using Bayesian Evidential Learning. Water Resources Research, in press, http://dx.doi.org/10.1002/2017WR022135.

780 Jesußek, A., Grandel, S., Dahmke, A., 2013. Impacts of subsurface heat storage on aquifer 781 hydrogeochemistry. Environ. Earth Sci., 69(6), 1999-2012, http://dx.doi.org/10.1007/s12665-012$782 \quad 2037-9$.

783 Kemna, A., 2000, Tomographic inversion of complex resistivity: theory and application. PhD Thesis. 784 University of Bochum.

785 Klepikova, M., Wildemeersch, S., Hermans, T., Jamin, P., Orban, P., Nguyen, F., Brouyère, S. 786 Dassargues, A., 2016. Heat tracer test in an alluvial aquifer: field experiment and inverse modelling. 787 J. Hydrol., 540, 812-823, http://dx.doi.org/10.1016/j.jhydrol.2016.06.066. 
788 Koestel J., Kemna A., Javaux M., Binley A., Vereecken H. 2008. Quantitative imaging of solute 789 transport in an unsaturated and undisturbed soil monolith with 3D ERT and TDR. Water Resour. 790 Res., 44, W12411, http://dx.doi.org/0.1029/2007WR006755.

791 LaBrecque D.J., Miletto M., Daily W., Ramirez A., Owen E., 1996. The effects of noise on Occam's 792 inversion of resistivity tomography data. Geophysics, 61(2), 538-548, 793 http://dx.doi.org/10.1190/1.1443980.

794 LaBrecque, D.J., Yang, X. 2001. Difference inversion of ERT data: a fast inversion method for 3-D 795 in-situ monitoring: J. Environ. Eng. Geoph., 6(2), 83-89, http://dx.doi.org/10.4133/JEEG6.2.83.

796 Lesparre, N., Nguyen, F., Kemna, A., Robert, T., Hermans, T., Daoudi, M., Flores-Orozco, A., 2017. 797 A new approach for time-lapse data weighting in ERT. Geophysics, 82(6), 1-35, 798 http://dx.doi.org/10.1190/geo2017-0024.1.

799 Liang, J., Yang, Q., Liu, L., Li, X., 2011. Modeling and performance evaluation of shallow ground 800 water heat pumps in Beijing plain, China. Energy Buildings, 43, 3131-3138, 801 http://dx.doi.org/10.106/j.enbuild.2011.08.007.

802 Lo Russo, S., Civita, M.V., 2009. Open-loop groundwater heat pumps development for large 803 buildings: a case study. Geothermics 38, 335-345, 804 http://dx.doi.org/10.1016/j.geothermics.2008.12.009.

805 Lo Russo, S. L., Boffa, C., Civita, M. V., 2009. Low-enthalpy geothermal energy: an opportunity to 806 meet increasing energy needs and reduce $\mathrm{CO} 2$ and atmospheric pollutant emissions in Piemonte, 807 Italy. Geothermics, 38(2), 254-262, http://dx.doi.org/10.1016/j.geothermics.2008.07.005.

808 Lo Russo, S. L., Gnavi, L., Roccia, E., Taddia, G., Verda, V., 2014. Groundwater Heat Pump 809 (GWHP) system modeling and Thermal Affected Zone (TAZ) prediction reliability: Influence of 810 temporal variations in flow discharge and injection temperature. Geothermics, 51, 103-112, 811 http://dx.doi.org/10.1016/j.geothermics.2012.02.001.

812 Mattsson, N., Steinmann, G., Laloui, L., 2008. Advanced compact device for the in situ determination 813 of geothermal characteristics of soils. Energy Buildings, 40, 1344-1352, 814 http://dx.doi.org/10.1016/j.enbuild.2007.12.003. 
815 Milnes, E., Perrochet, P., 2013. Assessing the impact of thermal feedback and recycling in open-loop 816 groundwater heat pump (GWHP) systems: a complementary design tool. Hydrogeol. J., 21(2), 505817 514, http://dx.doi.org/10.1007/s10040-012-0902-y.

818 Nguyen, F., Kemna, A., Robert, T., \& Hermans, T., 2016. Data-driven selection of the minimum819 gradient support parameter in time-lapse focused electric imaging. Geophysics, 81(1), A1-A5, 820 http://dx.doi.org/10.1190/GEO2015-0226.1.

821 Nimmer, R.E., Osiensky, J.L., Binley, A.M., Williams, B.C., 2008. Three-dimensional effects 822 causing artifacts in two-dimensional, cross-borehole, electrical imaging. J. Hydrol., 359(1), 59-70, 823 http://dx.doi.org/10.1016/j.jhydrol.2008.06.022.

824 Pollock, D., Cirpka, O.A., 2012. Fully coupled hydrogeophysical inversion of a laboratory salt tracer 825 experiment monitored by electrical resistivity tomography. Water Resour. Res., 48(1), 826 http://dx.doi.org/10.1029/2011WR010779.

827 Polydorides, N., Lionheart, W.R., 2002. A Matlab toolkit for three-dimensional electrical impedance 828 tomography: a contribution to the Electrical Impedance and Diffuse Optical Reconstruction Software 829 project. Meas. Sci. Technol., 13(12), 1871, http://dx.doi.org/10.1088/0957-0233/13/12/310.

830 Possemiers, M., Huysmans, M., Batelaan, O., 2015. Application of multiple-point geostatistics to 831 simulate the effect of small-scale aquifer heterogeneity on the efficiency of aquifer thermal energy 832 storage. Hydrogeology Journal 23, 971-981, http://dx.doi.org/10.1007/s10040-015-1244-3.

833 Raymond, J., Therrien, R., Gosselin, L., Lefebvre, R., 2011. A review of thermal response test 834 analysis using pumping test concepts. Ground Water, 49, 932-945, http://dx.doi.org/10.1111/j.1745835 6584.2010.00791.x.

836 Rein, A., Hoffmann, R., Dietrich, P., 2004. Influence of natural time-dependent variations of 837 electrical conductivity on DC resistivity measurements. J. Hydrol., 285(1), 215-232, 838 http://dx.doi.org/10.1016/j.jhydrol.2003.08.015.

839 Revil, A., Cathles, L.M., Losh, S., Nunn, J.A., 1998. Electrical conductivity in shaly sands with 840 geophysical applications. J. Geophys. Res., 103(B10), 23925-23936, 841 http://dx.doi.org/10.1029/98jb02125.

842 Rucker, D.F., 2014. Investigating motion blur and temporal aliasing from time-lapse electrical 843 resistivity: J. Appl. Geophys., 111, 1-13, http://dx.doi.org/10.1016/j.jappgeo.2014.09.010. 
844 Sarbu, I., Sebarchievici, C., 2014. General review of ground-source heat pump systems for heating 845 and cooling of buildings. Energy and buildings, 70, 441-454, http://dx.doi.org/10.5772/61372.

846 Singha, K., Moysey, S., 2006. Accounting for spatially variable resolution in electrical resistivity 847 tomography through field-scale rock-physics relations. Geophysics, 71(4), A25-A, 848 http://dx.doi.org/2810.1190/1.2209753.

849 Singha, K., Day-Lewis, F.D., Johnson, T., Slater, L.D., 2015. Advances in interpretation of 850 subsurface processes with time-lapse electrical imaging. Hydrol. Proc., 29(6), 1549-1576, 851 http://dx.doi.org/10.1002/hyp.10280.

852 Slater L., Binley, A.M., Daily W., Johnson, R., 2000. Cross-hole electrical imaging of a controlled 853 saline tracer injection. J. Appl. Geophys., 44, 85-102, http://dx.doi.org/10.1016/S0926854 9851(00)00002-1.

855 Sommer, W., Valstar, J., van Gaans, P., Grotenhuis, T., Rijnaarts, H., 2013. The impact of aquifer 856 heterogeneity on the performance of aquifer thermal energy storage: Water Resources Research 49, 857 8128-8138, http://dx.doi.org/10.1002/2013WR013677.

858 Sommer, W.T., Doornenbal, P.J., Drijver, B.C., van Gaans, P.F.M., Leusbrock, I., Grotenhuis, J.T.C., 859 Rijnaarts, H.H.M., 2014. Thermal performance and heat transport in aquifer thermal energy storage. 860 Hydrogeology Journal 22, 263-279, http://dx.doi.org/10.1007/s10040-013-1066-0.

861 Therrien, R., McLaren, R.G., Sudicky, E.A., Panday, S.M., 2010. HydroGeoSphere: a three862 dimensional numerical model describing fully-integrated subsurface and surface flow and solute 863 transport. Groundwater Simulations Group, University of Waterloo, Waterloo, ON.

864 Tikhonov, A. 1963. Solution of incorrectly formulated problems and the regularization method. 865 Soviet Meth. Dokl., 4, 1035-1038.

866 Van Hoorde, M., Hermans, T., Dumont, G., Nguyen, F. 2017. 3D electrical resistivity tomography of 867 karstified formations using cross-line measurements. Engineering Geology, 230, 123-132, 868 http://dx.doi.org/10.1016/j.enggeo.2017.01.028.

869 Vanhoudt, D., Desmedt, J., Van Bael, J., Robeyn, N., Hoes, H., 2011. An aquifer thermal storage 870 system in a Belgian hospital: Long-term experimental evaluation of energy and cost savings. Energy 871 and Buildings, 43(12), 3657-3665, http://dx.doi.org/10.1016/j.enbuild.2011.09.040. 
872 Voigt, H.D., Haefner, F., 1987. Heat transfer in aquifers with finite caprock thickness during a thermal 873 injection process. Water Resour. Res., 2286-2292, 874 http://dx.doi.org/10.1029/wr023i012p02286.

875 Wildemeersch, S., Jamin, P., Orban, P., Hermans, T., Klepikova, M., Nguyen, F., Brouyère, S., 876 Dassargues, A., 2014. Coupling heat and chemical tracer experiments for estimating heat transfer 877 parameters in shallow alluvial aquifers. J. Contam. Hydrol., 169, 90-99, 878 http://dx.doi.org/10.1016/j.jconhyd.2014.08.001. 